\title{
Cyclin-dependent kinase 5 modulates the P2X2a receptor channel gating through phosphorylation of C-terminal threonine 372
}

Article in Pain · July 2017

DOI: 10.1097/j.jpain.0000000000001021

\section{CITATION}

1

12 authors, including:

Claudio Coddou

Universidad Católica del Norte (Chile), Coquimbo, Chile 32 PUBLICATIONS 793 CITATIONS

SEE PROFILE

Patricio Castro

University of Concepción

17 PUbLICATIONS 117 CITATIONS

SEE PROFILE

\section{READS}

71

2 Rodrigo Sandoval

University of Chile

11 PUBLICATIONS 29 CITATIONS

SEE PROFILE

Pablo Lazcano

University of Chile

3 PUBLICATIONS 10 CITATIONS

SEE PROFILE

Some of the authors of this publication are also working on these related projects:

Role of EPAC in neuronal differentiation View project

SILENCING OF CDK5 AS GENE THERAPY FOR NEURODEGENERATIVE DISEASES View project 


\title{
Cyclin-dependent kinase 5 modulates the P2X2a receptor channel gating through phosphorylation of C-terminal threonine 372
}

Claudio Coddou, ${ }^{a, b}$, Rodrigo Sandoval ${ }^{c}$, Patricio Castro ${ }^{a}$, Pablo Lazcano ${ }^{c}$, Maria José Hevia ${ }^{a}$, Milos Rokic ${ }^{b}$, Bradford Hall $^{d}$, Anita Terse ${ }^{d}$, Christian Gonzalez-Billaulte, f,g , Ashok B. Kulkarni ${ }^{d}$, Stanko S. Stojilkovic ${ }^{b}$, Elias Utreras ${ }^{\mathrm{c}, *}$

\begin{abstract}
The purinergic P2X2 receptor (P2X2R) is an adenosine triphosphate-gated ion channel widely expressed in the nervous system. Here, we identified a putative cyclin-dependent kinase 5 (Cdk5) phosphorylation site in the full-size variant P2X2aR $\left({ }^{372} \mathrm{TPKH}^{375}\right)$, which is absent in the splice variant P2X2bR. We therefore investigated the effects of Cdk5 and its neuronal activator, p35, on P2X2aR function. We found an interaction between P2X2aR and Cdk5/p35 by co-immunofluorescence and co-immunoprecipitation in HEK293 cells. We also found that threonine phosphorylation was significantly increased in HEK293 cells co-expressing P2X2aR and p35 as compared to cells expressing only P2X2aR. Moreover, P2X2aR-derived peptides encompassing the Cdk5 consensus motif were phosphorylated by Cdk5/p35. Whole-cell patch-clamp recordings indicated a delay in development of use-dependent desensitization (UDD) of P2X2aR but not of P2X2bR in HEK293 cells co-expressing P2X2aR and p35. In Xenopus oocytes, P2X2aRs showed a slower UDD than in HEK293 cells and Cdk5 activation prevented this effect. A similar effect was found in P2X2a/3R heteromeric currents in HEK293 cells. The P2X2aR-T372A mutant was resistant to UDD. In endogenous cells, we observed similar distribution between P2X2R and Cdk5/p35 by co-localization using immunofluorescence in primary culture of nociceptive neurons. Moreover, co-immunoprecipitation experiments showed an interaction between Cdk5 and P2X2R in mouse trigeminal ganglia. Finally, endogenous P2X2aR-mediated currents in PC12 cells and P2X2/3R mediated increases of intracellular $\mathrm{Ca}^{2+}$ in trigeminal neurons were Cdk5 dependent, since inhibition with roscovitine accelerated the desensitization kinetics of these responses. These results indicate that the P2X2aR is a novel target for Cdk5-mediated phosphorylation, which might play important physiological roles including pain signaling.
\end{abstract}

Keywords: P2X2R, Cdk5 activity, Phosphorylation, Patch-clamp current, Trigeminal ganglia, P2XR

\section{Introduction}

The cyclin-dependent kinase 5 (Cdk5) is an emergent and essential kinase involved in the physiology of sensory pathways. ${ }^{41,52} \mathrm{Cdk5}$ plays key roles in brain development and function, including neuronal migration, membrane transport, axon guidance, and pain

Sponsorships or competing interests that may be relevant to content are disclosed at the end of this article.

a Department of Biomedical Sciences, Faculty of Medicine, Universidad Católica del Norte, Coquimbo, Chile, ${ }^{b}$ Section on Cellular Signaling, The Eunice Kennedy Shiver National Institute of Health and Human Development, National Institutes of Health, Bethesda, MD, USA, ${ }^{\circ}$ Laboratory of Molecular and Cellular Mechanisms of Pain, Department of Biology, Faculty of Science, Universidad de Chile, Santiago, Chile, ${ }^{d}$ Functional Genomics Section, Laboratory of Cell and Developmental Biology, National Institute of Dental and Craniofacial Research, National Institutes of Health, Bethesda, MD, USA, ${ }^{\ominus}$ Laboratory of Cellular and Neuronal Dynamics, Department of Biology, Faculty of Science, Universidad de Chile, Santiago, Chile, ${ }^{f}$ Center for Geroscience, Brain Health and Metabolism (GERO), Santiago, Chile, ${ }^{g}$ The Buck Institute for Research on Aging, Novato, CA, USA

${ }^{*}$ Corresponding author. Address: Department of Biology, Faculty of

Sciences, Universidad de Chile, Las Palmeras 3425, Nuñoa, Santiago, 7800003 Chile.Tel.: 56-2-29787423; fax: 56-2-22712983. E-mail address: elias.utreras@ uchile.cl (E. Utreras).

PAIN 00 (2017) 1-14

(C) 2017 International Association for the Study of Pain

http://dx.doi.org/10.1097/j.pain.0000000000001021 signaling. ${ }^{19,20,52}$ Although Cdk5 is ubiquitously expressed, it is mostly active in postmitotic neurons, where its specific activators p35 and p39 are mainly expressed. ${ }^{19,30,55}$ Cdk5 is a prolinedirected serine/threonine kinase that phosphorylates many proteins, including cytoskeletal proteins, ion channels, and regulatory proteins. ${ }^{2}$ We reported earlier that Cdk5 regulates pain signaling in nociceptive neurons of the dorsal root ganglion (DRG) and trigeminal ganglion (TG). ${ }^{41,46,47,52}$ The Cdk5 activity increases during peripheral inflammation, by the action of cytokines such as TNF- $\alpha{ }^{47,53,57}$ and TGF- $\beta 1 .{ }^{54,56}$ Moreover, we found that Cdk5 phosphorylates the transient receptor potential vanilloid 1 (TRPV1) in Thr407, a key ion channel implicated in pain, increasing its function. ${ }^{25,33,40,47,54,56}$ Remarkably, TRPV1 is phosphorylated in Ser116 and Thr370 by PKA, ${ }^{35}$ and its dephosphorylation is important for the desensitization process. ${ }^{36}$ Interestingly, other channels such as the purinergic P2X receptors (P2XRs) also contribute to pain signaling. However, their functional modulation by Cdk5 has not been deeply analyzed.

The P2XRs are a family of extracellular adenosine triphosphate (ATP)-gated ionic channels, widely expressed in different tissues and involved in several physiological and pathophysiological processes. ${ }^{7,49}$ The P2X2R is one of the most abundant subtypes in the nervous system and its subunits can form homomeric or heteromeric channels with P2X3R subunit (P2X2/3R), which are 
expressed in nociceptive neurons and their activation is involved in pain signaling. ${ }^{8,9}$ Several functional splice variants of P2X2R exist, ${ }^{13}$ that differ in their desensitization properties; eg, the shorter variant P2X2bR lacks 69 amino acids in the intracellular Cterminal domain and desensitizes faster than the full-length P2X2aR. ${ }^{5,28,48}$ Compared to other P2XR subtypes, P2X2Rs exhibit slow desensitization profile, which depends on agonist and $\mathrm{Ca}^{2+}$ concentration, and in $\mathrm{N}$ - and $\mathrm{C}$-terminal residues/ domains. ${ }^{13,14,27}$ The gating properties of P2X2R are also affected by protons, divalent metals, ${ }^{11,34}$ reactive oxygen species, ${ }^{12}$ steroid hormones, ${ }^{18}$ and membrane phosphoinositides, ${ }^{22}$ all these molecules act as allosteric regulators. Additionally, phosphorylation of P2X2R by PKA and PKC also regulates its gating properties. $^{3,16}$

Here, we show that Cdk5 regulates the activity of P2X2R, by phosphorylation of Thr372, a residue exclusively present in the full-length P2X2aR but absent in P2X2bR. Using confocal microscopy, biochemical and electrophysiological techniques, we found that Cdk5/p35 physically interacts with $\mathrm{P} 2 \mathrm{X} 2 \mathrm{R}$ in heterologous overexpression systems, primary cultures of TG and DRG neurons, and in PC12 cells that endogenously express Cdk5/p35 and P2X2R. We also found that $\mathrm{Cdk5/p35}$ can phosphorylate P2X2aR, regulating the gating properties that results in desensitization changes, in both heterologous and endogenous systems. These findings suggest that the P2X2aR is a new target for Cdk5-mediated phosphorylation, which might play an important role in pain signaling and therefore could be a potential novel target for developing pain therapies.

\section{Methods}

\section{1. $P 2 X 2 R$ and $P 2 X 2 / 3 R$ expression in HEK293 cells and Xenopus laevis oocytes}

HEK293 cells (ATCC\# CRL-1573) were grown in Dulbecco modified Eagle medium containing 10\% of fetal bovine serum (FBS) and penicillin/streptomycin (Invitrogen, Carlsbad, CA). For Western blot and co-immunoprecipitation analysis, HEK293 cells were transiently co-transfected during 24 hours with rat P2X2aR-pIRES-GFP or P2X2bR-pIRES-GFP in combination with mouse p35 and mouse Cdk5 vectors by using Lipofectamine 2000 reagent (Invitrogen). For immunofluorescence experiments, HEK293 cells were grown on 12$\mathrm{mm}$ coverslips treated with poly-L-lysine and then transfected for 24 hours with rat P2X2aR-GFP fusion protein, mouse p35, and Cdk5 plasmids. For patch-clamp experiments, HEK293 cells were grown on 35-mm dishes at a density of 500,000 cells per plate and transfected with rat P2X2aR-pIRES-GFP, P2X2bR-pIRES-GFP, or P2X2aR-pIRES-GFP T372A mutant receptor with or without p35 vectors. For the experiments with the heteromeric P2X2/3R, we co-transfected rat P2X2aRpIRES-GFP, rat P2X3R-pIRES-GFP, and p35 vector, and for the isolation of the heteromeric currents, we used the specific agonist $\alpha, \beta$-methylene ATP ( $\alpha, \beta$-meATP). For experiments in Xenopus laevis oocytes, a segment of the ovary was surgically removed from female frogs under anesthesia with benzocaine $0.05 \%$ and oocytes were manually defolliculated and incubated with collagenase $\|(1 \mathrm{mg} / \mathrm{mL})$. Each oocyte was injected intranuclearly with 3 to $5 \mathrm{ng}$ of complementary DNA coding for the different P2X2Rs alone or combined with the p35 plasmid. Patch-clamp experiments for HEK293 cells and 2-electrode voltage-clamp experiments for Xenopus oocytes were performed 18 to 48 hours after transfection/injection.

\subsection{Primary culture of neurons from mouse TG and DRG and rat TG}

Nociceptive neurons were cultured as described previously. ${ }^{47}$ Briefly, TG and DRG were dissected out from 2- to 4-month-old mice or rat and incubated with collagenase $X I(0.66 \mathrm{mg} / \mathrm{mL})$ and dispase II (3 mg/mL) (Sigma-Aldrich, San Louis, MO) in an INCmix solution ( $\mathrm{NaCl} 155$ mM; $\mathrm{K}_{2} \mathrm{HPO}_{4} 1.5 \mathrm{mM}$; HEPES $10 \mathrm{mM}$; glucose $5 \mathrm{mM}$; at $\mathrm{pH}$ 7.4). Enzymatic digestion was performed for 45 minutes at $37^{\circ} \mathrm{C}$ in $5 \% \mathrm{CO}_{2}$, and cells were cultured in minimum essential media (MEM) supplemented with 10\% FBS, penicillin/streptomycin $100 \mathrm{mg} / \mathrm{mL}$, and MEM-vit (Invitrogen). Cells were plated on 12-mm poly-L-lysine-coated glass coverslips and cultured for 2 days. Animal experiments were conducted in accordance with the principles and procedures of the National Institutes of Health Guidelines for the Care and Use of Laboratory and the Bioethical Committee of Universidad Católica del Norte, Coquimbo, and the Ethics Committee of the Biology Department, Faculty of Sciences, Universidad of Chile, Santiago, Chile.

\subsection{PC12 cell culture}

The PC12 cell line (ATCC\# CRL-1721) was grown in Dulbecco modified Eagle medium containing 10\% FBS, 5\% horse serum, and penicillin/streptomycin. For immunofluorescence and electrophysiological recordings experiments, PC12 cells were plated on 12-mm coverslips treated with poly-L-lysine. For Cdk5 inhibition experiment, PC12 cells were treated with roscovitine $30 \mu \mathrm{M}$ during 6 and 24 hours before electrophysiological recordings. To evaluate the current of P2X2aR or P2X2aR T372A mutant, we transfected those vectors in PC12 cells and current was measured 24 hours later.

\subsection{Western blot analysis}

Protein extracts from HEK293 cells, PC12 cells, or from mouse TG and DRG were obtained in T-PER buffer (Pierce, Rockford, IL) with Complete Mini protease inhibitor cocktail tablets and PhosSTOP phosphatase inhibitor cocktail tablets (Roche Diagnostic, Indianapolis, IN). Protein concentration was determined using the Bradford Protein Assay (Bio-Rad, Hercules, CA). Proteins were separated in sodium dodecyl sulfate polyacrylamide gel electrophoresis gels and transferred to nitrocellulose membranes (Invitrogen). Membranes were soaked in blocking buffer (5\% nonfat dry milk in Tris-buffered saline with 0.05\% Tween-20 for 1 hour at room temperature and then incubated overnight at $4^{\circ} \mathrm{C}$, with primary antibody diluted in $1 \%$ nonfat dry milk blocking buffer). The membranes were washed in Tris-buffered saline with $0.05 \%$ Tween-20 and incubated for 1 hour at room temperature with the secondary antibodies diluted in $1 \%$ nonfat dry milk blocking buffer. Immunoreactivity was detected by using Super-Signal West Pico or Dura Chemiluminescent Substrate (Thermo Scientific, Rockford, IL). Western blots were performed using P2X2R rabbit antibody \#APR-003 and P2X3R rabbit antibody \#APR-016 from Alomone Labs (Jerusalem, Israel); Cdk5 rabbit antibody C8, Cdk5 mouse antibody DC17, p35 rabbit antibody C19, and p35 goat antibody A18 from Santa Cruz Biotechnology (Dallas, TX); phospho-(Thr) MAPK/CDK substrate mouse antibody \#2321, GFP rabbit antibody \#2555, and p35 \#2680 were obtained from Cell Signaling Technology; and $\alpha$-tubulin antibody from SigmaAldrich was used as loading control. We also used a control peptide antigen from $\mathrm{P} 2 \mathrm{X} 2 \mathrm{R}$ sequence (Alomone Labs) as 
negative control for P2X2R Western blot and immunofluorescence experiments. The optical densities of the bands were quantified using an image analysis system with ImageJ 1.46r software (NIH, Bethesda, MD).

\subsection{Co-immunoprecipitate assay}

To evaluate the putative interaction between P2X2aR and Cdk5/ p35, we immunoprecipitated protein (IP) extracts $(1 \mathrm{mg})$ from HEK293 cells co-transfected with P2X2aR-IRES-GFP and p35; or P2X2aR-IRES-GFP and Cdk5 plasmids, by using with $2 \mu \mathrm{g}$ of rabbit p35 antibody (C19) or mouse Cdk5 antibody (DC17), respectively. In addition, we immunoprecipitated $350 \mu \mathrm{g}$ of protein extracts from mouse TG by using $2 \mu \mathrm{g}$ of rabbit Cdk5 (C8) antibody. The incubation of antibodies was performed for 4 hours at $4^{\circ} \mathrm{C}$ with stirring followed by the addition of $25 \mu \mathrm{L}$ of Protein A/G PLUS-agarose beads (Santa Cruz Biotechnology) incubated overnight at $4^{\circ} \mathrm{C}$ with stirring. Immunoprecipitated proteins were washed by centrifugation at $2500 \mathrm{rpm}$ at $4^{\circ} \mathrm{C} 3$ times in cold phosphate-buffered saline (PBS). Loading buffer was added to IPs and they were electrophoresed on sodium dodecyl sulfate polyacrylamide gel electrophoresis (protocol continues as Western blot analysis). To evaluate threonine phosphorylation of $\mathrm{P} 2 \mathrm{X} 2 \mathrm{aR}$ mediated by Cdk5/p35, we immunoprecipitated $300 \mu \mathrm{g}$ of protein extracts from HEK293 cells co-transfected with P2X2aR-IRES-GFP with or without p35 plasmid by using $2 \mu \mathrm{g}$ of P2X2R rabbit antibody \#APR-003 from Alomone Labs and followed a Western blot against phospho-(Thr) MAPK/CDK substrate mouse antibody \#2321. We used normal IgG goat antibody (\#AB-108-C; R\&D System, Minneapolis, MN) as IgG control. As negative control, we used protein extracts from HEK293 cells overexpressing P2X2aR and p35 or Cdk5 incubated only with Protein A/G PLUS-agarose beads without primary antibody.

\subsection{Immunofluorescence analysis}

We analyzed the co-localization of P2X2aR with Cdk5/p35 and the cellular localization of P2X2aR-T372A in HEK293 cells cotransfected with those vectors. Additionally, we analyzed the P2X2R and p35 endogen expression in PC12 cells. Furthermore, we examined the co-localization of endogen P2X2R and P2X3R, with $\mathrm{p} 35$ and Cdk5 in primary culture of neurons from mouse TG and DRG. Thus, transfected HEK293 cells, PC12 cells, or primary cultured neurons were washed with warm PBS for 5 minutes and fixed with a $4 \%$ PFA-4\% sucrose solution in PBS at $37^{\circ} \mathrm{C}$ for 20 minutes. After fixation, cells were washed 3 times with PBS and permeabilized for 5 minutes with a $0.2 \%$ Triton X-100 solution in PBS. After washout with PBS, cells were blocked with a 5\% BSA solution in PBS at room temperature for 1 hour. Corresponding primary antibodies were used at following concentrations: antiCdk5 rabbit C8 (1:100) and anti-Cdk5 mouse DC17 (1:100) antibody (Santa Cruz Biotechnology); anti-p35 goat A18 (1:100) and anti-p35 rabbit C19 (1:100) antibody (Santa Cruz Biotechnology); MAP1B goat N-19 (1:200) antibody (Santa Cruz Biotechnology); anti-slll tubulin mouse clone G7121 (1:1000) antibody (Promega); and P2X2R rabbit \#APR-003 (1:250) antibody (Alomone Labs). All primary antibodies were diluted in $1 \%$ BSA solution and incubated overnight at $4^{\circ} \mathrm{C}$. The coverslips were washed with PBS and then incubated for 1 hour at room temperature with corresponding secondary antibodies: antirabbit conjugated to Alexa Fluor546, anti-mouse conjugated to Alexa Fluor488, and anti-goat conjugated to Alexa Fluor647 (Molecular Probes; Life Technologies, Grand Island, NY) in combination with Dapi (Thermo Fisher Scientific). Finally, coverslips were washed with PBS, then they were dried and mounted on a slide with FluorSave (Calbiochem) and then were observed using confocal microscopy (LSM 710 Meta Model; Carl Zeiss Microscopy) and processed with the LSM Image Browser (Carl Zeiss Microscopy) software.

\subsection{Preparation of RNA and reverse transcription polymerase chain reaction}

Conventional reverse transcription polymerase chain reaction (RT-PCR) was performed as described previously. ${ }^{47}$ Briefly, total RNA was extracted from frozen DRGs and TGs from wild-type 5month-old mice or from PC12 cells by using TRlzol Reagent (Invitrogen) according to the manufacturer's instructions. Total RNA was digested with TURBO DNase (Ambion, Austin, TX) for 1 hour to remove contaminating genomic DNA, and retrotranscription was carried out with M-MLV Reverse Transcriptase (Invitrogen) by using Oligo (dT). We analyzed messenger RNA levels of $\mathrm{p} 35$, Cdk5, P2X2R isoforms, P2X3R, and S29 by using conventional RT-PCR. The following primers were used: p35 sense (S): 5'-GCC CTT CCT GGT AGA GAG CTG-3', p35 antisense (AS): 5'-GTG TGA AAT AGT GTG GGT CGG C-3'; Cdk5 S: 5'-GGC TAA AAA CCG GGA AAC TC-3', Cdk5 AS: 5'CCA TTG CAG CTG TCG AAA TA-3'; P2X2R common S: 5' -GGA ACT GTG ACC TGG ACT TG-3', P2X2R common AS: 5'-CAG ATC CAG GTC TGT AGC TT-3'; P2X2aR S: 5'-CAA GGC ACC CCT CAA GTA GA-3', P2X2aR AS: 5' -GCT GGT CCT GGG AGT AGT GA-3'; P2X3R S: 5'-CAG GGC ACT TCT GTC TाT GTC-3', P2X3R AS: 5'-AGC GGT ACT TCT CCT CAT TCT C-3'; S29 S: 5'-GGA GTC ACC CAC GGA AGT TCG G-3' and S29 AS: 5'-GGA AGC ACT GGC GGC ACA TG-3'.

\subsection{In vitro phosphorylation of $P 2 X 2 a R$-based peptides}

We identified a putative consensus motif for C $\mathrm{dk} 5$ phosphorylation in P2X2aR protein sequence by using prediction websites Motif-Scan http://scansite.mit.edu/motifscan_seq.phtml and NetPhos2.0 http://www.cbs.dtu.dk/services/NetPhos/. To evaluate potential Cdk5 phosphorylation sites, 10 amino acid residues of $\mathrm{P} 2 \mathrm{X} 2 \mathrm{aR}$ peptides were tested in an in vitro Cdk5 kinase assay. Recombinant full-length human Cdk5 and p35 (Sigma) were incubated in $50 \mu \mathrm{L}$ of kinase assay buffer $(100 \mathrm{mM}$ Tris-HCl [pH 7.4]; $50 \mathrm{mM} \mathrm{MgCl}_{2} ; 5$ mM EDTA; $50 \mu \mathrm{M} \mathrm{NaF} ; 5 \mu \mathrm{M}$ $\mathrm{Na}_{2} \mathrm{VO}_{3}$; and $5 \mathrm{mM}$ DTT) containing either $10 \mu \mathrm{g}$ of histone $\mathrm{H} 1$ (Sigma) or P2X2aR peptides (mouse P2X2aR, KVRTPRHPSS; human P2X2aR, KVCTPSHPSG; rat P2X2aR, KVRTPKHPSS; and nonrelated peptide, IGQSPFHGDD). Kinase assays were carried out at $30^{\circ} \mathrm{C}$ for 60 minutes by adding $5 \mu \mathrm{Ci}$ of $\left[\gamma^{32} \mathrm{P}\right]$ ATP $(0.5 \mathrm{mM})$. The peptide assay was stopped by adding 10\% trichloroacetic acid to precipitate proteins. Twenty-microliter aliquots of trichloroacetic acid supernatant were transferred onto P81 phosphocellulose squares (spotted in duplicates), air-dried, and washed 5 times for 15 minutes each in $75 \mathrm{mM}$ phosphoric acid and once in 95\% ethanol. After air drying, squares were transferred to vials containing Bio-Safe II scintillation fluid (Research Products International, Mount Prospect, IL) for counting in a Beckman Coulter (Fullerton, CA) scintillation counter (model SL 3801).

\subsection{Current measurements in HEK 293 and PC12 cells}

Electrophysiological experiments were performed on cells at room temperature using whole-cell patch-clamp recording 
techniques. The currents were recorded using an Axopatch 200B patch-clamp amplifier (Molecular Devices) and were filtered at 2 $\mathrm{kHz}$ using a low-pass Bessel filter. Patch electrodes, fabricated from borosilicate glass (type 1B150F-3; World Precision Instruments), using a Flaming Brown horizontal puller (P-87; Sutter Instruments), were heat polished to a final tip resistance of 2 to 4 $\mathrm{M} \Omega$. All current records were captured and stored using the pClamp 9 software packages in conjunction with the Digidata 1322A analog-to-digital converter (Molecular Devices). Unless otherwise specified, patch electrodes were filled with a solution containing the following: $142 \mathrm{mM} \mathrm{NaCl}, 10 \mathrm{mM}$ EGTA, and $10 \mathrm{mM}$ HEPES. The osmolarity of the internal solutions was 305 mOsm. The bath solution (KR-like) contained the following: $142 \mathrm{mM} \mathrm{NaCl}, 3 \mathrm{mM} \mathrm{KCl}, 1 \mathrm{mM} \mathrm{MgCl}_{2}, 2 \mathrm{mM} \mathrm{CaCl}, 10 \mathrm{mM}$ glucose, and $10 \mathrm{mM}$ HEPES. The $\mathrm{Ca}^{2+}$-free solution contained $145 \mathrm{mM} \mathrm{NaCl}, 3 \mathrm{mM} \mathrm{KCl}, 10 \mathrm{mM}$ glucose, and $10 \mathrm{mM}$ HEPES. In all cases, $\mathrm{pH}$ was adjusted to 7.35 , and the osmolarity of those solutions was 295 to $305 \mathrm{mOsm}$. Adenosine triphosphate was daily prepared in bath buffer and applied using a rapid solution changer system (RSC-200; Biologic Science Instruments). The current responses were recorded from single cells clamped at $60 \mathrm{mV}$. Most of the data were collected from recordings of single cells stimulated with $10 \mu \mathrm{M}$ of ATP with a washout interval of 4 minutes between each application and normalized to the highest current amplitude. For PC12 recordings of endogenous P2X2Rmediated currents, we used similar conditions to that used for HEK293 cells recordings, but the intracellular solution contained $5 \mathrm{mM}$ ATP and $0.05 \mathrm{mM}$ EGTA to observe the endogenous phosphorylation of the P2X2R by Cdk5. Roscovitine ( $30 \mu \mathrm{M})$ was incubated for 6 and 24 hours before experiments.

\subsection{Current measurements in Xenopus oocytes}

Oocytes were bathed in Barth solution $(88 \mathrm{mM} \mathrm{NaCl}, 1 \mathrm{mM} \mathrm{KCl}$, $2.4 \mathrm{mM} \mathrm{NaHCO}_{3}, 10 \mathrm{mM} \mathrm{HEPES}, 0.82 \mathrm{mM} \mathrm{MgSO}_{4}, 0.33 \mathrm{mM} \mathrm{Ca}$ $\left(\mathrm{NO}_{3}\right)_{2}$, and $0.91 \mathrm{mM} \mathrm{CaCl}_{2}, \mathrm{pH}$ 7.5) supplemented with $10 \mathrm{IU} / \mathrm{L}$ penicillin/10 mg streptomycin and $2 \mathrm{mM}$ pyruvate; oocytes were clamped at $-60 \mathrm{mV}$ using the 2-electrode voltage-clamp configuration with an OC-725C clamper (Warner Instruments). The ATP-evoked currents were recorded after regular 1 to $1000 \mu \mathrm{M}$ ATP applications, with washout periods ranging from 3 to 15 minutes depending on ATP concentration. For the experiments with allosteric modulators, oocytes expressing P2X2aR or P2X2aR-T372A vectors were challenged with $10 \mu \mathrm{M}$ ATP that resulted in small, non-desensitizing currents. Copper, zinc, and PPADS solutions (selective P2XR antagonist) at a concentration of $10 \mu \mathrm{M}$ were pre-applied for 1 minute and then were co-applied with ATP; the currents induced by this application were compared to that induced by ATP alone. For the $\mathrm{pH}$ experiments, the ATP-containing solution was adjusted to $\mathrm{pH}$ 6.5 and the currents induced by this solution were compared to the currents induced by ATP at pH 7.5.

\subsection{Intracellular $\mathrm{Ca}^{2+}$ measurements in cultured TG neurons}

Primary cultured TG neurons were loaded with Fluo4-AM ( $3 \mu \mathrm{M}$ in DMSO; Molecular Probes, Eugene, OR) for 30 minutes at $37^{\circ} \mathrm{C}$. The cells were then washed twice with PBS and incubated for 30 minutes at $37^{\circ} \mathrm{C}$, and they were mounted in a perfusion chamber that was placed on the stage of a confocal microscope (Zeiss LSM800) at 22 to $24^{\circ} \mathrm{C}$. Purinergic agonists (ATP or $\alpha, \beta$-metilATP) were added at the corresponding time at a final concentration of $100 \mu \mathrm{M}$ in bath solution. Cultured cells were briefly illuminated adjusting laser power $(\leq 0.5 \%)$ and scan speed (6-7), using the ZEN 2.1 software (Zeiss). Regions of interest were simultaneously selected on neuronal somata containing Fluo-4 fluorescence (laser excitation $480 \mathrm{~nm}$, emission $510 \mathrm{~nm}$ ) in a field having usually more than 20 cells. Images were collected at 2-second intervals during a continuous 12-minute period. Calcium imaging was carried out in a 16-bit (0-65,000 units of fluorescence scale) with highly sensitive GaAsP detector (Zeiss). The $\mathrm{Ca}^{2+}$ transients, defined by their amplitude that should be greater than 2 times the noise level, were acquired and analyzed offline with ZEN 2.1 software.

\subsection{Statistical analysis}

All experiments were performed a minimum of 3 times. All graphs show the average \pm SEM. Statistical evaluation was performed with GraphPad Prism software, version 6.1 (GraphPad, San Diego, CA). Significant differences between experiments were assessed by an unpaired $t$ test or a 1-way analysis of variance with a Tukey posttest, considering $\alpha$ as 0.05 . For electrophysiological experiments, statistical analyses were performed using the nonparametric Mann-Whitney test.

\section{Results}

\subsection{The $P 2 X 2 a R$ binds to the Cdk5/p35 complex in heterologous expression system}

To study the potential P2X2aR modulation by Cdk5, we first evaluated whether there is an interaction between the P2X2aR and the Cdk5/p35 complex in HEK293 cells co-transfected with Cdk5, $\mathrm{p} 35$, and P2X2aR vectors. For this end, we transfected P2X2aRGFP fusion vector (Fig. 1A) and found that GFP fluorescence from P2X2aR-GFP fusion protein (green) was located mainly on the plasma membrane and it was highly co-localized with P2X2R label (red), allowing us to use GFP fluorescence as a measure of P2X2aR localization (Fig. 1B). To confirm the specificity of P2X2R antibody, we used a control peptide antigen for P2X2R that was incubated with P2X2R primary antibody, demonstrating that P2X2R label almost disappears, while GFP fluorescence remains (Fig. 1C). Next, by immunofluorescence using Cdk5 and p35 antibodies, we found that P2X2aR-GFP (green) co-localized with Cdk5 (red) and p35 (blue) immunostaining in close proximity to plasma membrane (Fig. 1D). In addition, we performed a set of confocal images at different focal planes (0.9 $\mu \mathrm{m}$ each) (Fig. 1E) and an orthogonal reconstruction (Fig. 1F) to find evidence that supports that P2X2aR-GFP, Cdk5, and p35 are interacting with each other, and they were effectively found co-localized in areas near to the plasma membrane. To confirm the specificity of P2X2R antibody in Western blot analysis, we incubated the P2X2R primary antibody in the presence or absence of control peptide antigen, and we observed that the P2X2R bands disappeared in the presence of the peptide in both heterologous expression of $\mathrm{P} 2 \mathrm{X} 2 \mathrm{aR}$ transfected HEK293 cells or in endogenous P2X2R expression in PC12 cells (Fig. 2A). We also evaluated whether P2X2aR can immunoprecipitate with Cdk5 or p35 in HEK293 cells cotransfected with P2X2aR-pIRES-GFP with p35 or Cdk5 vectors. By immunofluorescence, we observed that P2X2aR expression was localized in plasma membrane and GFP fluorescence was localized in the whole cell (Fig. 2B). We performed coimmunoprecipitation experiments using HEK293 cells under the following experimental condition: (1) control untransfected cells (UT); (2) cells transfected with P2X2aR-pIRES-GFP vector; (3) cells transfected with P2X2aR-pIRES-GFP plus p35 vectors; and (4) cells transfected with P2X2aR-pIRES-GFP plus His-Cdk5 vectors, 

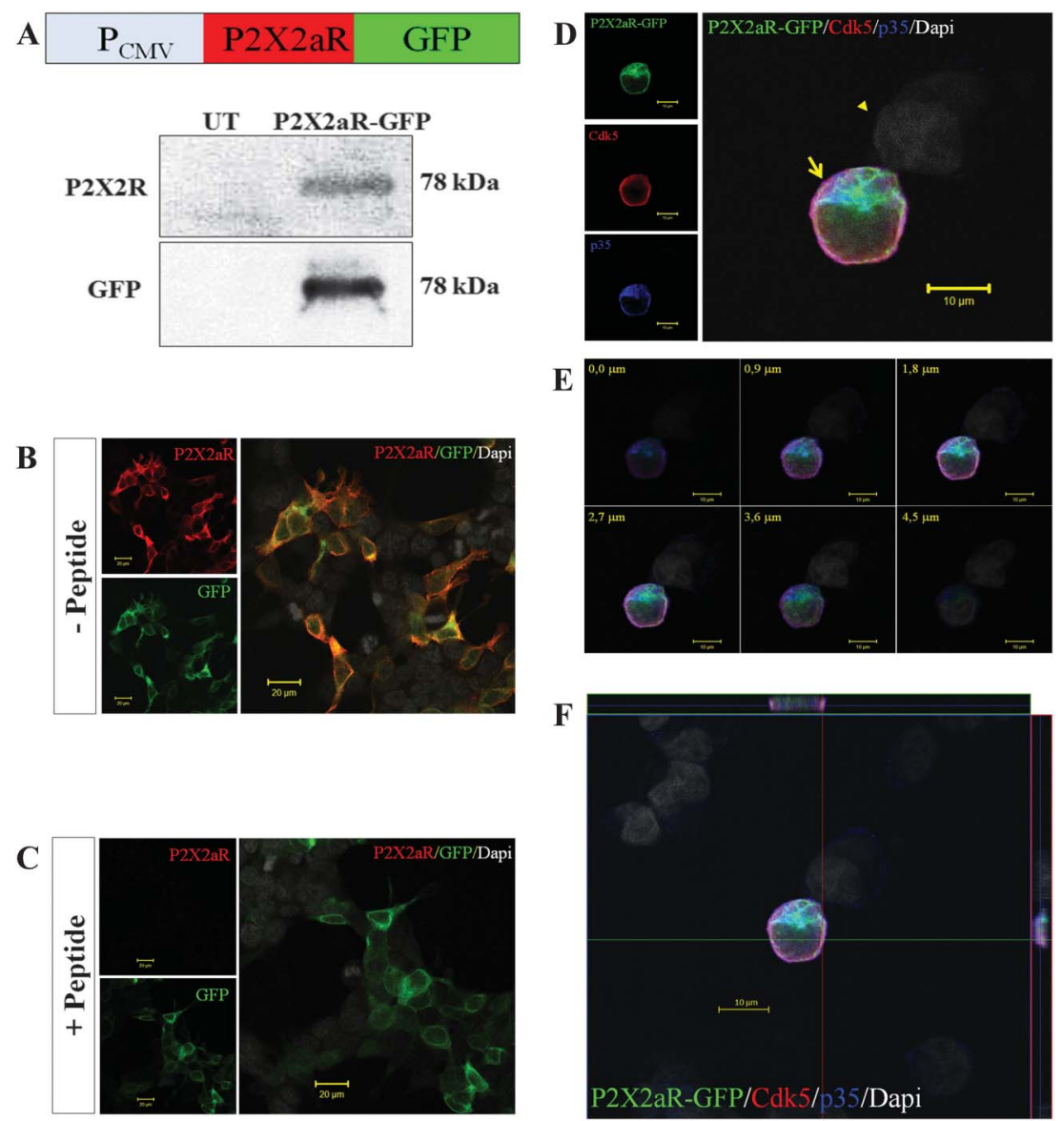

Figure 1. Co-localization of P2X2aR with Cdk5/p35 in HEK293 cells. (A) Upper panel, scheme of P2X2aR-GFP vector used for transfection of HEK293 cells. Lower panel, Western blot for P2X2R and GFP from HEK293 cells transfected with P2X2aR-GFP vector or untransfected (UT). (B and C) Confocal images of HEK293 cells expressing P2X2aR-GFP vector in the absence (B) or in the presence (C) of control peptide antigen for P2X2R. GFP fluorescence from P2X2aR-GFP fusion protein (green) was located mainly in the plasma membrane and it highly co-localizes with P2X2R label (red). Dapi (white) was used for nuclear staining. (D) Confocal immunofluorescence of HEK293 cells expressing P2X2aR-GFP, Cdk5, and p35 vectors (arrow) and untransfected HEK293 (arrow head). P2X2aR-GFP green fluorescence was observed co-localizing with Cdk5 (red) and p35 (blue) immunostaining, both close to plasma membrane. (E) Set of 6 confocal images at different focal planes (0.9 $\mu \mathrm{m}$ each) showing co-localization of P2X2aR-GFP with Cdk5/p35. (F) Orthogonal analysis reveals a high co-localization of P2X2aR-GFP, Cdk5, and p35 in region near to plasma membrane of triple transfected HEK293 cells. Cdk5, cyclin-dependent kinase 5.

and then we confirmed the expression of transfected proteins through Western blots (Fig. 2C). Using protein extracts from HEK293 cells co-transfected with P2X2aR and p35, or from P2X2aR and His-Cdk5, we immunoprecipitated by using an antip35 antibody (Fig. 2D) or anti-Cdk5 antibody (Fig. 2E). In the immunocomplexes from both immunoprecipitates, we detected the P2X2aR in Western blot using an anti-P2X2R antibody. As a control of immunoprecipitation, we detected the corresponding Cdk5 and p35 proteins after stripping each membrane. Moreover, as controls for immunoprecipitation assays, we used goat lgG control and no primary antibody (only Protein AVG agarose beads) (Fig. 2D, E). These results indicate that $\mathrm{P} 2 \mathrm{X} 2 \mathrm{aR}$ form a protein complex with Cdk5 and p35 in co-transfected HEK293 cells. The formation of such complex could be independent of mutual presence of Cdk5 and p35. This finding prompted us to investigate if there is a link between the activities of Cdk5 signaling pathway and P2X2aR.

\subsection{The $P 2 X 2 a R$ is a target for Cdk5 phosphorylation}

By using the phosphorylation prediction websites Motif-Scan and NetPhos2.0, we identified a putative consensus motif for Cdk5 phosphorylation of $(\mathrm{S} / \mathrm{T}) \mathrm{PX}(\mathrm{K} / \mathrm{H} / \mathrm{R})$ in the $\mathrm{P} 2 \mathrm{X} 2 \mathrm{aR}$ protein sequence. ${ }^{2}$ Remarkably, this consensus motif ${ }^{372} \mathrm{TPKH}^{375}$ sequence is present exclusively in the full-length rat $\mathrm{P} 2 \mathrm{X} 2 \mathrm{aR}$ but absent in the shorter splice variant P2X2bR (Fig. 3A). By using P2X2R antibody, we recognized P2X2aR, P2X2bR, and P2X2aR-T372A protein transfected in HEK293 cells (Fig. 3B). We evaluated whether P2X2aR-Cdk5/p35 interactions result in the phosphorylation of threonine residues, an indication that Cdk5 could directly phosphorylate the P2X2aR. To evaluate this, we immunoprecipitated P2X2aR from transfected HEK293 cells with P2X2aR alone or with p35 vector, by using P2X2R antibody followed by Western blot against phosphothreonine antibody. We found that phosphothreonine signal was significantly increased in cells expressing P2X2aR and p35, as compared with P2X2aR alone (Fig. 3C). To further study whether this P2X2aR sequence is a phosphorylation target for Cdk5/p35, we generated 10-amino acid peptides containing the threonine Cdk5 motif present in rat, mouse, and human P2X2aRs (Fig. 3D) and tested their phosphorylation potential by an in vitro kinase assay by using recombinant Cdk5/ p35 protein. As a positive control, we used the phosphorylation of histone $\mathrm{H} 1$ as measured by the incorporation of ${ }^{32} \mathrm{P}$. We found that all 3 P2X2aR-derived peptides were highly phosphorylated by the recombinant Cdk5/p35 complex with 45.9\% for human P2X2aR, $56.3 \%$ for mouse $\mathrm{P} 2 \mathrm{X} 2 \mathrm{aR}$, and $64.0 \%$ for rat $\mathrm{P} 2 \mathrm{X} 2 \mathrm{aR}$ as compared with histone $\mathrm{H} 1$ phosphorylation (100\%). A nonrelated 


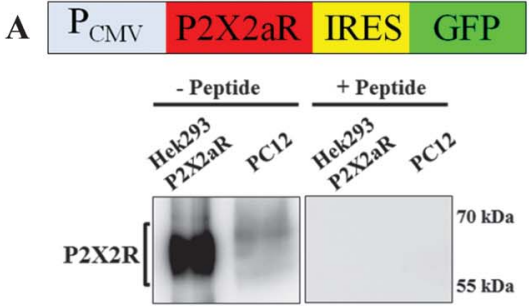

C

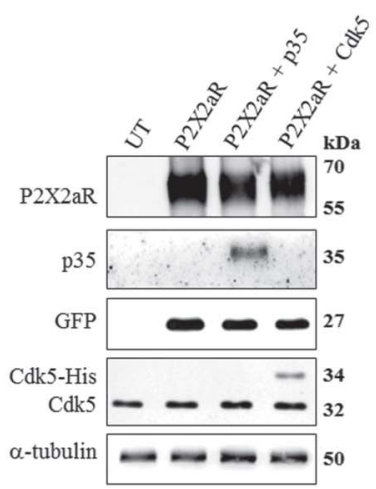

B

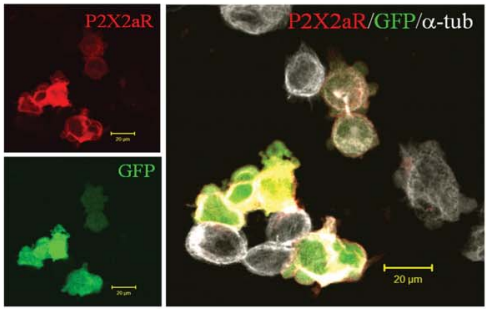

D

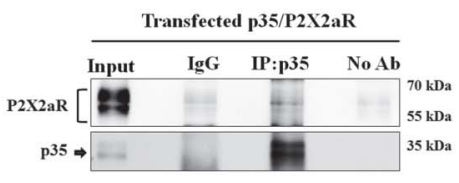

$\mathbf{E}$

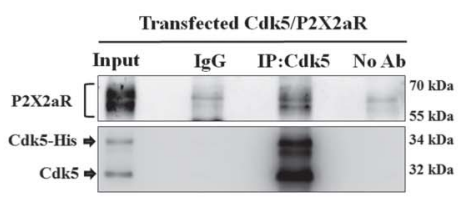

Figure 2. Co-immunoprecipitation of P2X2aR with Cdk5/p35 in HEK293 cells. (A) Upper panel, scheme of P2X2aR-IRES-GFP vector used for transfection of HEK293 cells. Lower panel, Western blot for P2X2R from HEK293 transfected or from PC12 in the absence or the presence of control peptide antigen for P2X2R. (B) Confocal images of HEK293 cells expressing P2X2aR-IRES-GFP vector. GFP fluorescence (green) was located in entire transfected cells; in contrast, P2X2aR immunostaining was mainly localized in the plasma membrane. $\alpha$-Tubulin ( $\alpha$-tub, white) was used as cellular staining. (C) Analysis of protein expression of HEK293 cells expressing P2X2aR-pIRES-GFP, His-Cdk5, and p35 vectors. (D and E) Immunoprecipitation experiments of HEK293 cells expressing P2X2aR-pIRES-GFP and p35; or P2X2aR-pIRES-GFP and Cdk5. For immunoprecipitation, we used p35 rabbit (C19) antibody (D) and Cdk5 mouse (DC17) antibody (E), and we detected P2X2aR by using P2X2R rabbit antibody by Western blot. As a positive control, the corresponding Cdk5 and p35 proteins were detected after stripping of each membrane. For a control with no antibody, we only used Protein A/G agarose beads and we did not find the P2X2aR in those immunocomplexes. We used normal lgG goat antibody as IgG control. Cdk5, cyclin-dependent kinase 5.

10-amino acid peptide was used as negative control for Cdk5 activity and it was 1 order of magnitude less phosphorylated (3.4\%) than P2X2aR peptides (Fig. 3D). These experiments also showed that in vitro phosphorylation of P2X2aR-derived peptides from human, rat, and mouse occurred at the same extent when using similar experimental conditions.

\subsection{Cdk5 activation regulates $P 2 X 2 a R$ desensitization}

We tested if this interaction between the P2X2aR and Cdk5/p35 has any functional effects. For this purpose, we studied the patterns of currents generated by P2X2aR activation using electrophysiological techniques and tested Cdk5-mediated changes in those currents. As we have previously demonstrated, the P2X2R has a unique property, an increase in receptor desensitization rate after repetitive ATP applications that is termed use-dependent desensitization (UDD). ${ }^{14,27}$ In HEK293 cells expressing the P2X2aR and measured under the whole-cell configuration, receptor desensitization increased after repetitive $10 \mu \mathrm{M}$ ATP applications in the presence of extracellular $\mathrm{Ca}^{2+}$ (Fig. 4A). Cells co-expressing the P2X2aR and p35 still showed UDD although the percentage of desensitization in the third and fourth ATP application was significantly smaller (Fig. $4 \mathrm{~A}, \mathrm{C}, \mathrm{n}=6, P<0.05)$. In contrast, no difference in the development of UDD was found between cells expressing the P2X2bR alone or co-expressed with p35 (Fig. 4B, D, $\mathrm{n}=6$ ). Activation of Cdk5 by transfection of p35 did not affect the current amplitudes of both receptors (Fig. 4E, F). Afterwards, we wanted to test these effects in Xenopus laevis oocytes, a different heterologous expression system. The increase in receptor desensitization in oocytes was significantly lower, and only after four $100 \mu \mathrm{M}$ ATP applications a modest increase in receptor desensitization was observed (Fig. 5A, B). However, co-expression of P2X2aR and p35 prevented the modest increase in desensitization observed at the fourth $100 \mu \mathrm{M}$ ATP application (Fig. 5A, B, E), suggesting a role of Cdk5 in this effect. No differences in ATP EC 50 (Fig. 5C) nor in maximal current (Fig. 5D) were observed when the P2X2aR was expressed alone or together with p35. Finally, treatment with the Cdk5 inhibitor roscovitine (30 $\mu \mathrm{M}$, incubated for 3 hours before the experiment) reverted the effect of Cdk5 activation and restored the desensitization observed in the P2X2aR alone (Fig. 5E). Thus, Cdk5-mediated phosphorylation should be responsible for P2X2aR desensitization.

To disrupt the putative Cdk5 phosphorylation site of the P2X2aR, ${ }^{372} \mathrm{TPKH}^{375}$, we generated the P2X2aR-T372A mutant. This mutant was successfully expressed in the plasma membrane of HEK293 cells and was indistinguishable from wild-type P2X2aR, as measured by immunofluorescence experiment (Fig. 6A, B). In addition, this mutant has similar molecular weight as compared with wild-type P2X2aR (Fig. 3B). In functional experiments, the whole-cell current elicited by ATP desensitized with an average $\tau_{\text {des }}$ of $10.3 \pm 1.2$ seconds, but no further increases in UDD were observed as compared to the wild-type P2X2aR in HEK293 transfected cells (Fig. 6C). Next, we overexpressed both the wild-type P2X2aR and the T372A mutant in PC12 cells during 24 hours, and we observed similar results to that obtained with HEK293 cells (Fig. 6D). Similar to what we observed for wild-type P2X2aR, when the T372A mutant was expressed in oocytes, the rates of receptor desensitization were slower than in HEK293 cells (Fig. 6E). In addition, ATP affinity was not significantly affected by this mutation ( $14.6 \pm 2.5 \mu \mathrm{M}$ in T372A vs $17.7 \pm$ $0.7 \mu \mathrm{M}$ in wild-type P2X2aR; Fig. $6 \mathrm{~F}$ ), and the maximal currents 
A

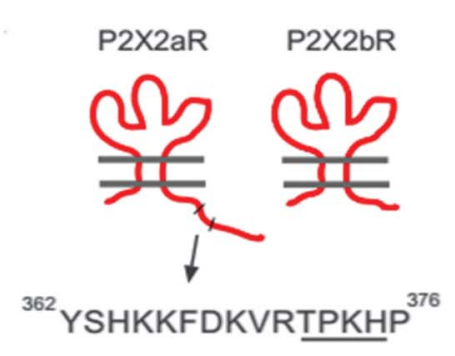

C
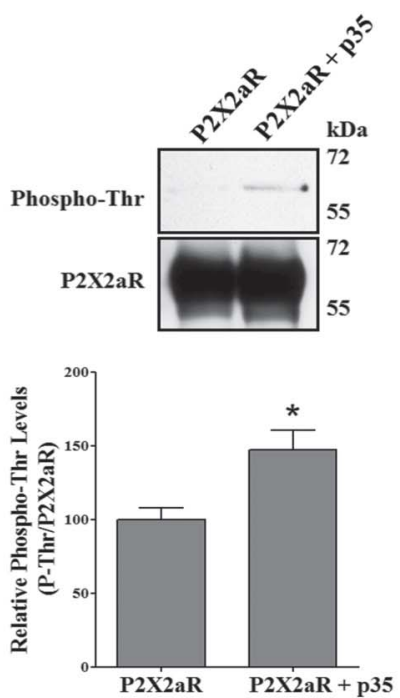

B

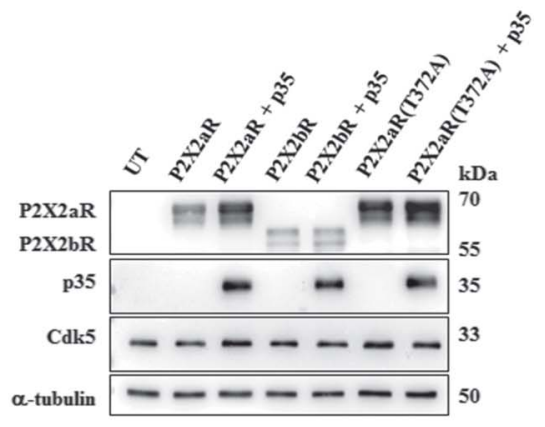

D P2X2aR Mouse P2X2aR Human P2X2aR Rat No related peptide
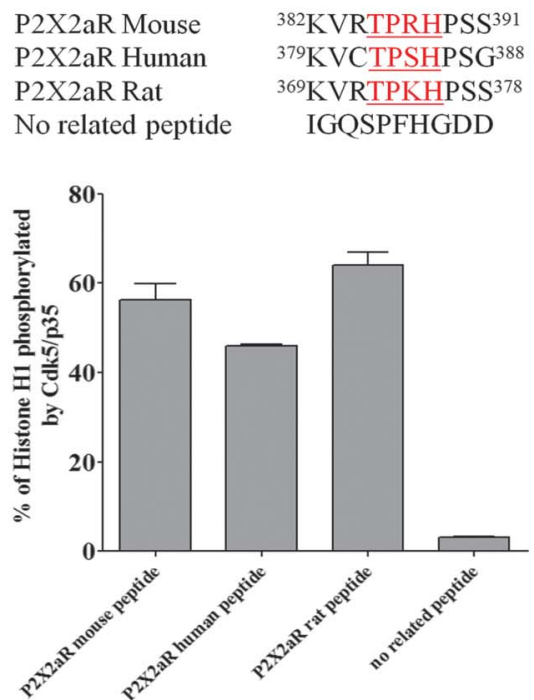

Figure 3. Cdk5/p35 phosphorylates the P2X2aR. (A) Representative cartoon showing the difference between the full-length P2X2aR and the short-variant P2X2bR, highlighting the putative Cdk5 site on the P2X2aR. (B) Representative Western blots against P2X2R, p35, and Cdk5 of HEK293 cells transfected with P2X2aR, P2X2bR, and P2X2aR-T372A, in the presence or absence of p35. $\alpha$-Tubulin is a loading control. (C) Upper panel, representative immunoprecipitation experiment on HEK293 cells transfected with P2X2aR-pIRES-GFP alone or with p35. Cell lysates were immunoprecipitated with a P2X2R antibody and then revealed with a Phospho-Thr antibody that recognizes phosphorylation in Cdk5-protein targets. Lower panel, Quantification of the increase in Phospho-Thr levels in cells coexpressing P2X2aR and p35, calculated from a densitometry analysis. $n=3,{ }^{*} P<0.05, t$ test. (D) Upper panel, sequences of the synthetic peptides derived from P2X2aR from mouse, human, and rat that include a specific underlined Cdk5 consensus motif (red) and a sequence of a nonrelated peptide as negative control. Lower panel, in vitro phosphorylation mediated by Cdk5/p35 complex detected by ${ }^{32} \mathrm{P}$ incorporation into the synthetic peptides. Histone $\mathrm{H} 1$ was used as positive control of Cdk5/p35 kinase activity and a nonrelated peptide was used as a negative control. $\mathrm{n}=6$ measures for each reaction. Cdk5, cyclin-dependent kinase 5 .

were affected only in oocytes but not in HEK293 cells (Fig. 6G). We hypothesize that the mutation induces important structural changes that affects the receptor gating, rendering a lessdesensitizing phenotype and preventing the effect of Colk5 activation as observed in wild-type receptors. To confirm that the T372A mutation did not alter other features of P2X2aR gating, we studied the effects of several allosteric modulators and one antagonist of this receptor. The typical potentiation of ATP-gated currents of P2X2aR induced by copper, zinc, and protons was conserved in the T372A mutant as well as the inhibition induced by PPADS (Fig. 6H). Finally, co-expression of the T372A mutant and p35 did not affect receptor desensitization in both HEK293 cells and oocytes (Fig. 6H). Thus, these results suggest that Cdk5-mediated phosphorylation of the P2X2aR at Thr372 can mainly affect the desensitization of this receptor channel.

\subsection{P2X2R interacts with Cdk5/p35 complex in nociceptive mouse tissues}

To evaluate whether P2X2R is also interacting with Cdk5 and p35 in relevant physiological tissues, we performed primary cultures of neurons from mouse DRG and TG. After 2 days of culture, by using immunofluorescence and confocal microscopy we detected that P2X2R (green) is expressed exclusively in a subset of TG (Fig. 7A, C) and DRG (Fig. 7B, D) neurons colocalizing with p35 (arrows, Fig. 7A, B) or Cdk5 (arrows, Fig. 7C, D). The expression of Cdk5 and p35 (red) was predominant in neurons, labeled with $\beta$ III-tubulin or Map1B antibodies (white). Cdk5 and p35 were also detected in other cell types present in our primary culture setup although showing reduced relative signal intensity (Fig. 7A-D). We also detected co-localization of P2X2R with p35 (Fig. 7E) or P2X2R with Cdk5 (Fig. 7F) in neurites from primary culture TG neurons. Moreover, by using RT-PCR, we detected that mouse $T G$ and $D R G$ expressed messenger RNA of P2X2R isoforms (P2X2aR, P2X2bR, and P2X2eR), P2X3R, Cdk5, and p35 (Fig. 7G). In addition, by using Western blot we detected several bands of P2X2R (between 55 and $72 \mathrm{kDa}$ ) from mouse TG and DRG tissues, according to splice variants and a different degree of glycosylation. ${ }^{39}$ We also detected P2X3R, Cdk5, and p35 by Western blot from these tissues (Fig. 7H). Finally, we immunoprecipitated extracts from mouse TG by using a rabbit anti-Cdk5 antibody and detected 
A

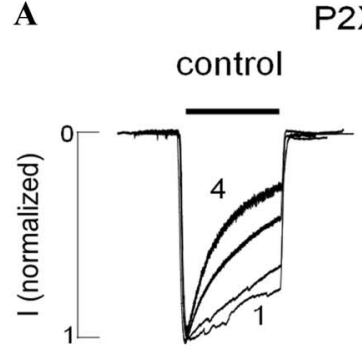

$\mathrm{P} 2 \mathrm{X} 2 \mathrm{aR}$

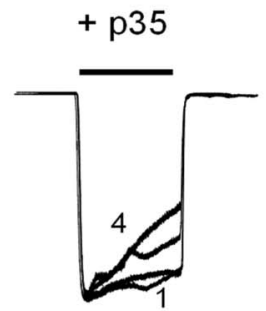

B

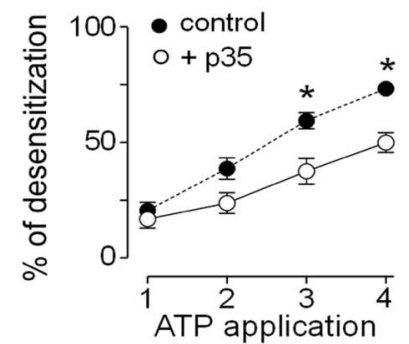

$\mathbf{E}$

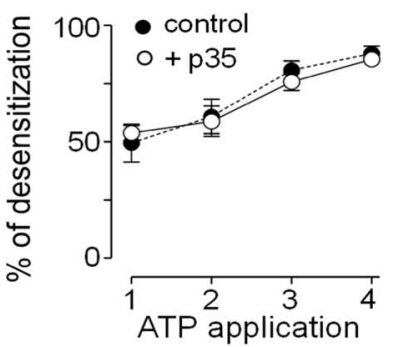

Figure 4. Cyclin-dependent kinase 5 (Cdk5) activation delays P2X2aR desensitization expressed in HEK293 cells. (A and D) Representative recordings of consecutive $10 \mu \mathrm{M}$ ATP applications in the presence of extracellular $\mathrm{Ca}^{2+}$, to single cells expressing the P2X2aR (A) or the P2X2bR (D) alone (left recordings) or coexpressed with p35 (right recordings). (B and E) Summary of the percentages of desensitization in cells expressing the P2X2aR (B) or the P2X2bR (E) alone (black circles) or co-expressed with p35 (open circles). ${ }^{*} P<0.05$, Mann-Whitney test, $\mathrm{n}=6$. (C and F) Current amplitudes evoked by $10 \mu \mathrm{M}$ ATP in HEK293 cells expressing the P2X2aR (C) or P2X2bR (F) alone or co-expressed with p35. $n=5$. ATP, adenosine triphosphate.

P2X2R in Western blot using a rabbit anti-P2X2R antibody. As a control for immunoprecipitation, we detected Cdk5 protein after stripping the membrane. As a control with no primary antibody, we only used Protein A/G agarose beads and we did not find P2X2R in those immunocomplexes (Fig. 7I). Altogether, these results indicate that endogen $\mathrm{P} 2 \mathrm{X} 2 \mathrm{R}$ interacts with $\mathrm{Cdk} 5$ in mouse TG and that P2X2R co-localize with Cdk5 and p35 in TG and DRG neurons, suggesting that Cdk5 might play an important role in physiological modulation of P2X2R, implicating its role in pain signaling.
$\mathbf{A}$

$\mathrm{P} 2 \times 2 \mathrm{aR}$

ATP $100 \mu \mathrm{M}, 40 \mathrm{~s}$

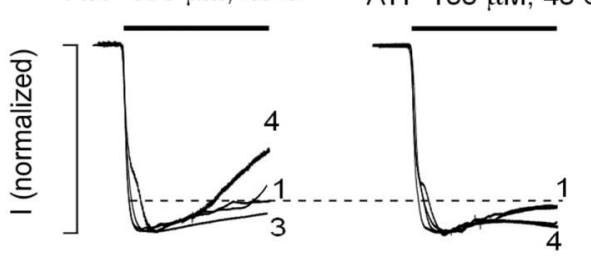

C

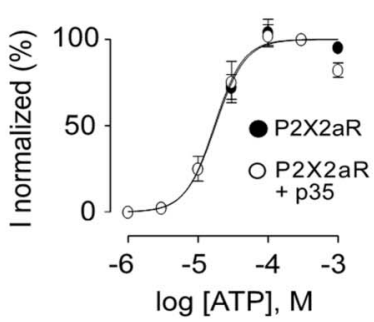

$\mathrm{P} 2 \times 2 \mathrm{aR}+\mathrm{p} 35$

D

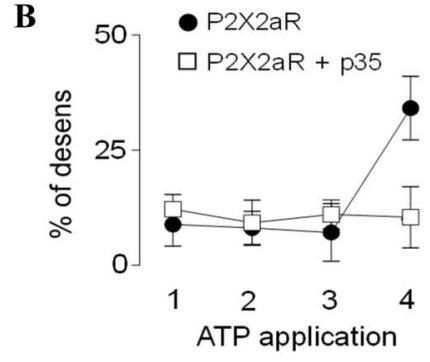

$\mathbf{E}$

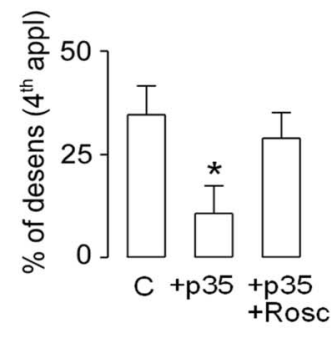

Figure 5. Effects of Cdk5 activation on the P2X2aR expressed in Xenopus oocytes. (A) Representative recordings of an oocyte expressing the P2X2aR alone (left recordings) or an oocyte co-expressing the receptor with p35 (right recordings). Oocytes were challenged with $100 \mu \mathrm{M}$ ATP and the first 40 seconds of applications are shown. In each case, 4 applications with 12-minute washouts are shown. (B) Summary of the percentages of desensitization after 4 consecutive $100 \mu$ M ATP applications. ${ }^{*} P<0.05$, Mann-Whitney test; $\mathrm{n}=5$. (C) Concentration-response curves for the P2X2aR alone (closed circles) or co-expressed with p35 (open circles). The estimated $\mathrm{EC}_{50}$ S were $17.7 \pm 0.7$ (•) and $17.1 \pm 1.9$ (0) $\mu \mathrm{M}, \mathrm{n}=5$. (D) Summary of current amplitude from oocytes expressing the P2X2aR alone (C) or together with p35 ( + p35); $n=7$. (E) Roscovitine (Rosc) treatment recovers the percentage of desensitization at the fourth ATP application observed in control oocytes (C) expressing the P2X2aR alone, $\mathrm{n}=4$ to 5. ${ }^{*} P<0.05$, Mann-Whitney test. ATP, adenosine triphosphate; Cdk5, cyclin-dependent kinase 5 . 


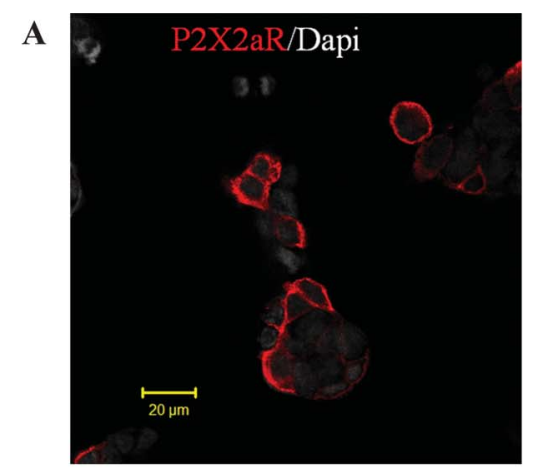

C

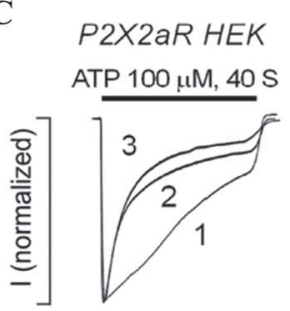

$\mathbf{E}$

T372A oocytes

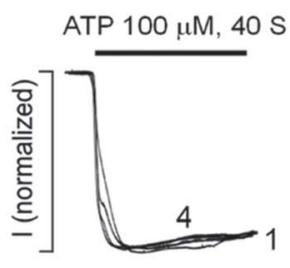

H

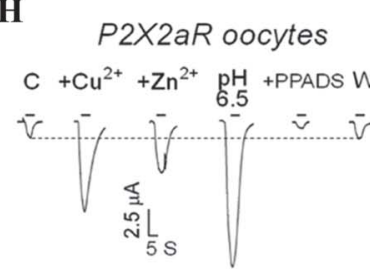

B

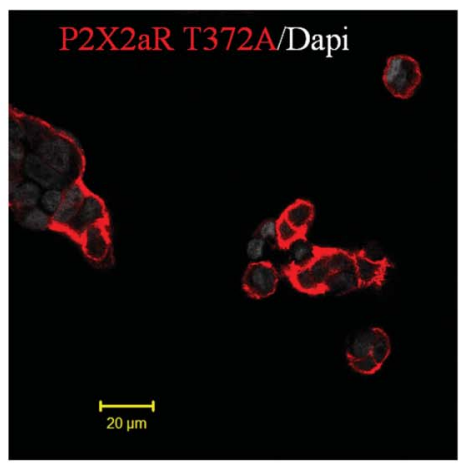

D

P2X2aR PC12 T372A PC12 ATP $100 \mu \mathrm{M}, 40 \mathrm{~S}$

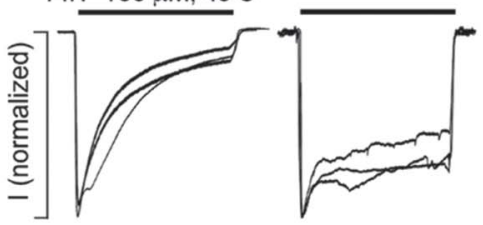

G

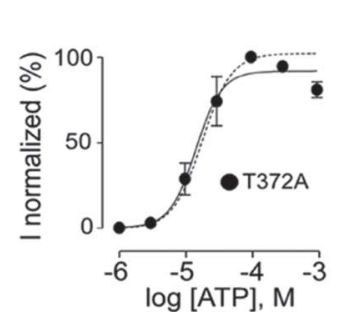

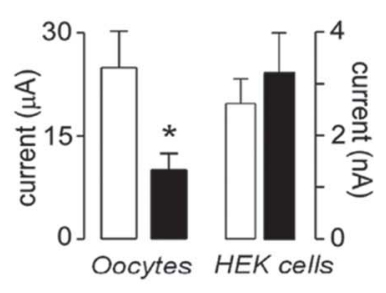
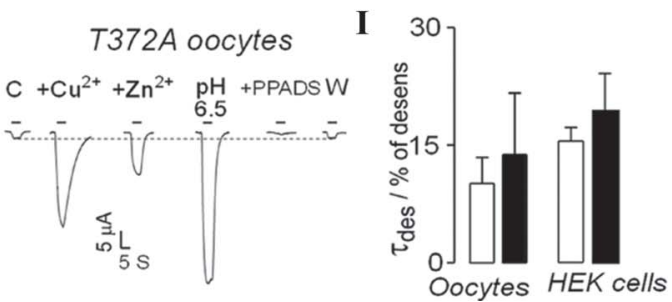

Figure 6. The mutant P2X2aR-T372A loses $\mathrm{Ca}^{2+}$-induced increase in desensitization. Immunofluorescence of HEK293 cells expressing the wild-type P2X2aR (A) or the T372A mutant (B) in red. Dapi (white) was used as nuclear staining. (C) Recordings from a HEK293 cell overexpressing the wild-type P2X2aR (left recording), showing the increase in desensitization after 4 consecutive $100 \mu \mathrm{M}$ ATP applications, or the T132A mutant (right recordings), showing no further increase in desensitization after repetitive ATP applications. (D) Recordings from a PC12 cell overexpressing the wild-type P2X2aR (left recordings) or the T132A mutant showing similar results to that obtained with HEK293 cells. (E) The same protocol in an oocyte expressing the T372A mutant and recorded with 2-electrode voltage-clamp technique. (F) The ATP concentration-response curve for the T372A mutant expressed in Xenopus oocytes. Dotted line represents the curve for the wild-type P2X2aR. $n=4$. (G) Maximal currents for the wild-type P2X2aR (open bars) and the T372A mutant (closed bars) obtained in oocytes and HEK293 cells. ${ }^{*} P<0.05$, Mann-Whitney test, $n=5$. (H) Allosteric modulation and inhibition of P2X2aR-mediated currents for the wild-type P2X2aR (left recordings) and the T132A mutant (right recordings). Ten micromolar $\mu$ of copper $\left(\mathrm{Cu}^{2+}\right)$, zinc $\left(\mathrm{Zn}^{2+}\right)$, or PPADS were pre-applied for 1 minute before its co-application with $10 \mu \mathrm{M}$ ATP; C = control (ATP alone), $\mathrm{W}=$ washout. Recordings are representative of 4 separate experiments. (I) Desensitization constants $\left(\tau_{\text {des }}\right)$ and percentage of desensitization (\% of desens) calculated for HEK293 cells and oocytes, respectively, expressing the T372A mutant alone (open bars) or co-expressed with the p35 (closed bars) ATP application; no significant differences in desensitization were observed, $n=4$ to 8 . ATP, adenosine triphosphate.

\subsection{Endogenous $P 2 X 2 R-m e d i a t e d$ currents are regulated by endogenous Cdk5 activity}

In a following set of experiments, we tested whether Cdk5 regulation could be relevant in a system that expresses both proteins endogenously by means of electrophysiology. We chose for this purpose neuronal-like PC12 cells, a cell line derived from rat pheochromocytoma that was the original source for the first P2X2R cloned $^{4}$ (Fig. 2A). These cells also endogenously express p35 and Cdk5. ${ }^{23,53}$ Undifferentiated PC12 cells exhibit ATP-mediated currents that match with the desensitization profile of the slow desensitizing P2X2aR ( $\tau_{\text {des }}=22.9 \pm 3.4$ seconds; Fig. 8A, B).
When cells were treated with roscovitine $(30 \mu \mathrm{M})$ for 6 or 24 hours to inhibit the endogenous Cdk5 activity, currents desensitized significantly faster $\left(\tau_{\text {des }} 6\right.$ hours $=7.1 \pm 2.5$ seconds; $\tau_{\text {des }}$ 24 hours $=9.0 \pm 2.0$ seconds; Fig. 8 A, B), without significantly altering the current densities (Fig. 8C), further confirming the role of Cdk5 in regulating the gating properties of the P2X2aR.

\subsection{The desensitization of the heteromeric $P 2 X 2 / 3 R$ is also modulated by Cdk5/p35}

In a final set of experiments, we tested the effects of Cdk5 on heteromeric P2X2/3Rs, which is the main purinergic subtype, 


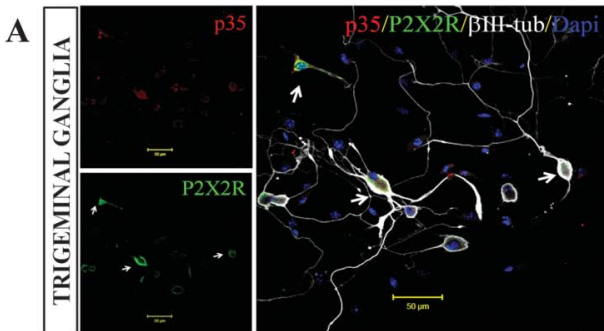

B
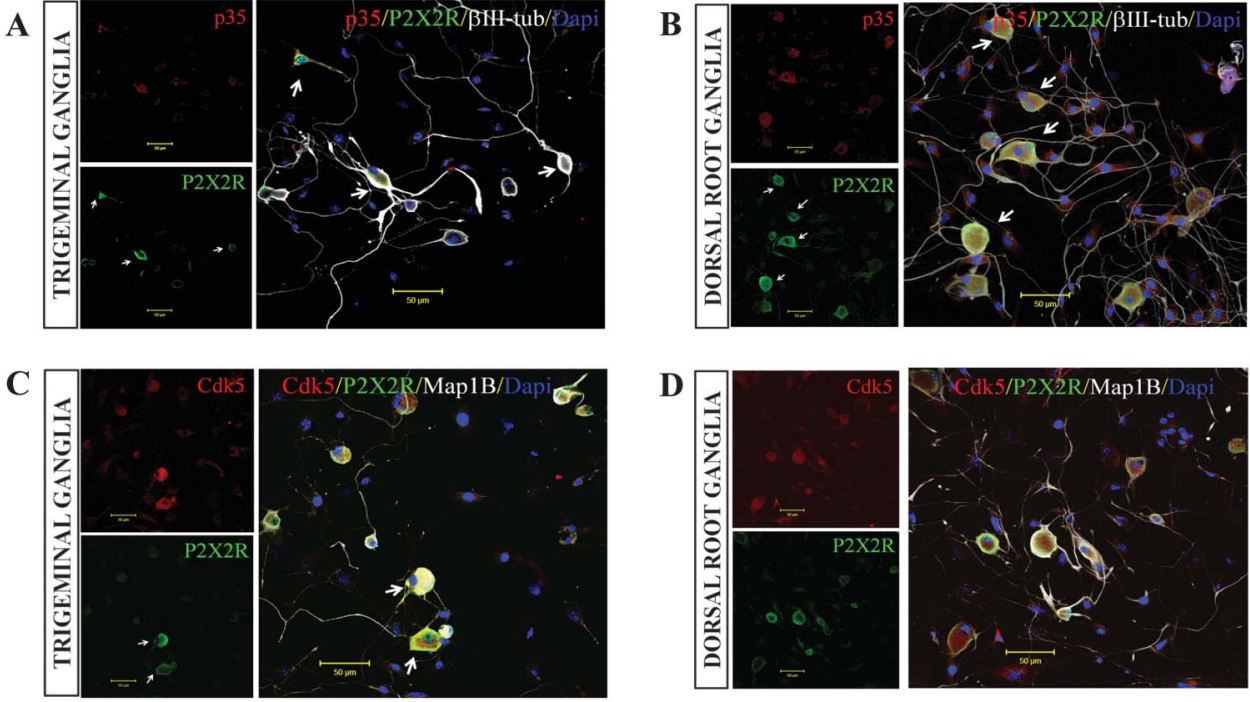

D

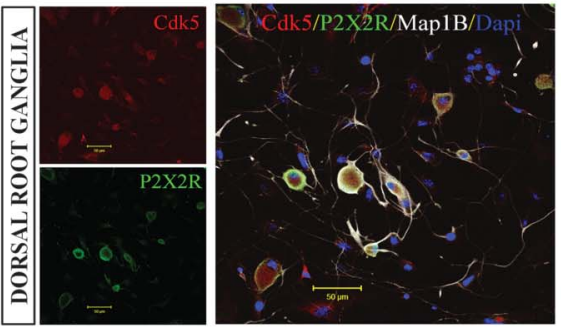

$\mathbf{E}$
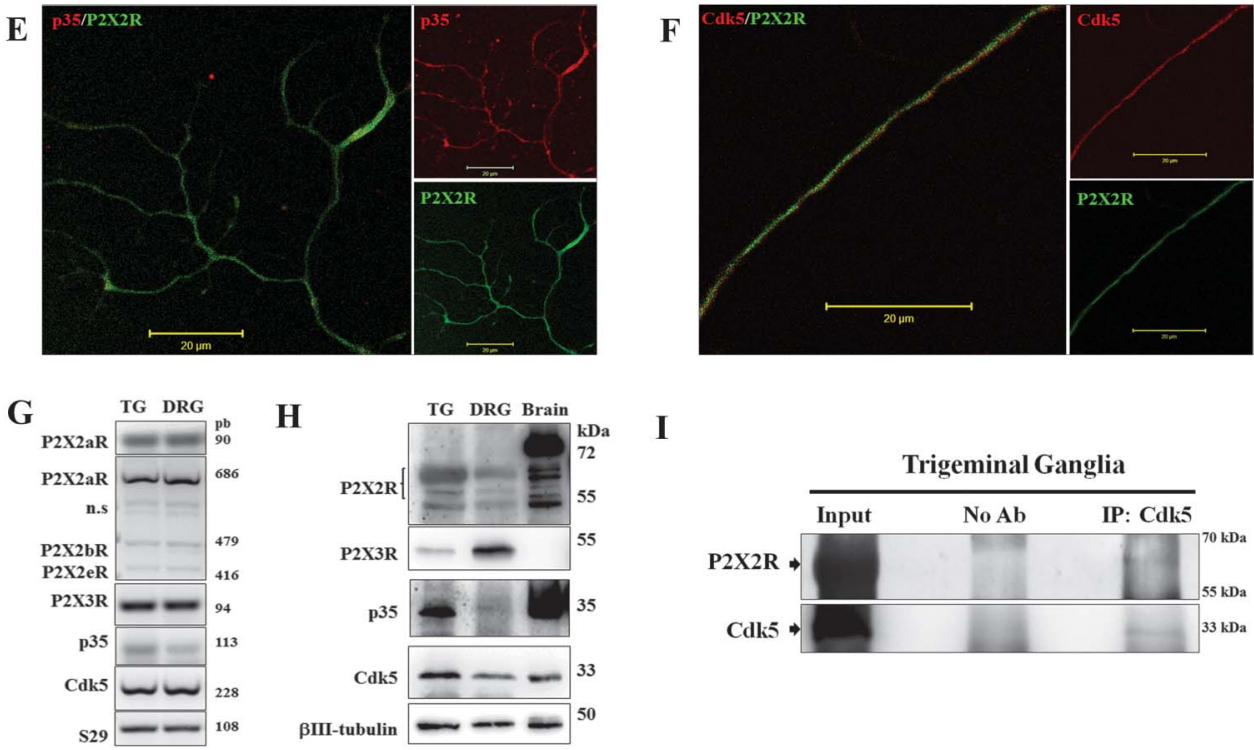

I

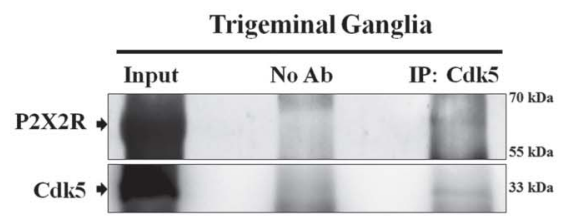

Figure 7. Interaction of P2X2R with Cdk5/p35 in mouse nociceptive neurons. Confocal immunofluorescence of primary cultured neurons of 2 DIV from mouse TG ( $A$ and $C$ ) and DRG (B and D), expressing endogenous levels of P2X2R (green), p35, and Cdk5 (red). Neurons were immunostained with $\beta$ III-tubulin or Map1B (white). Arrows show TG or DRG neurons positive for P2X2R staining. (E) Magnification of representative neurites from cultured TG neurons stained with P2X2R (green) and p35 (red). (F) Magnification of representative neurites from cultured TG neurons stained with P2X2R (green) and Cdk5 (red). (G) Representative RT-PCR from total RNA obtained from mouse TG and DRG tissues. P2X2aR, P2X2bR, P2X2eR, P2X3R, p35, and Cdk5 messenger RNA levels were evaluated. S29 levels were used as housekeeping control. $(H)$ Representative Western blots of protein extract from mouse TG, DRG, and brain tissues showing P2X2R, P2X3R, p35, Cdk5, and $\beta$ III-tubulin expression. (I) Immunoprecipitation experiments of mouse TG protein extracts with Cdk5 antibody and detected by Western blot for P2X2R. As a positive control, the corresponding Cdk5 bands were detected after stripping the membrane. For a control with no antibody, we only used Protein A/G agarose beads, and we did not find the P2X2R in those immunocomplexes. Cdk5, cyclin-dependent kinase 5; DRG, dorsal root ganglion; RT-PCR, reverse transcription polymerase chain reaction; TG, trigeminal ganglion.

expressed in pain pathways. In HEK293 cells co-transfected with the P2X2aR and the P2X3R, we evoked heteromeric currents by using the specific agonist $\alpha, \beta$-meATP that induces the typical slow desensitizing P2X2/3R-mediated currents (Fig. 8D); when p35 was co-transfected with both P2XRs, the resulting current desensitized slower than the one mediated by the P2X2/3R alone, with desensitization constants of $10.7 \pm 1.9$ and $5.8 \pm 0.8$ seconds, respectively (Fig. 8D, E), without significantly altering the current densities (Fig. 8F). To test the physiological relevance of this phenomenon, we first performed primary cultures of mouse TG (Fig. 9A) and DRG neurons (Fig. 9B) for 2 days and we observed co-localization of P2X2R (red) with P2X3R (green) by immunofluorescence. Later on, in acute primary cultures of rat TG, we measured the endogenous P2X2/ 3R-mediated responses by means of $\mathrm{Ca}^{2+}$ imaging. Adenosine triphosphate evoked strong responses of TG neurons, as reflected by the increases in fluorescence induced by ATP in Fluo-4 loaded neurons (Fig. 9C). Adenosine triphosphate, a general purinergic agonist, activated in average a $30 \%$ of the stimulated neurons (Fig. 9D). Interestingly, when $\alpha, \beta$-meATP was used, a P2X3R and P2X2/3R agonist, only an $8 \%$ of the TG neurons responded (Fig. 9D). The responding cells presented 2 main types of responses, a fast spike (black tracing), probably reflecting the activation of a homomeric P2X3R, and a slower spike (red tracing), that could reflect the activation of the P2X2/ 3R (Fig. 9E). Finally, when TG neurons were treated with 
A

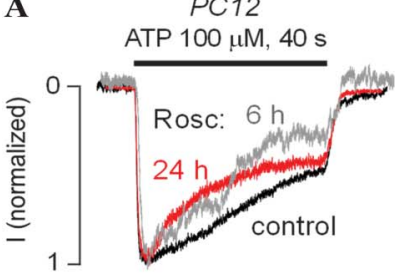

D

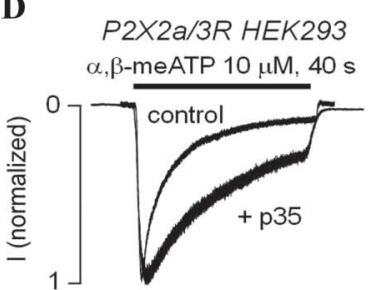

B

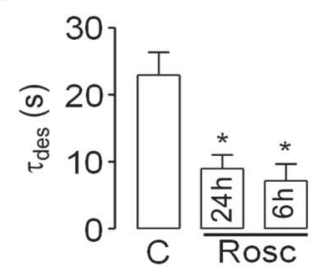

$\mathbf{E}$

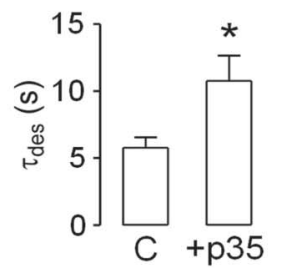

C

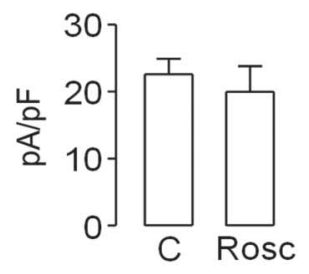

F

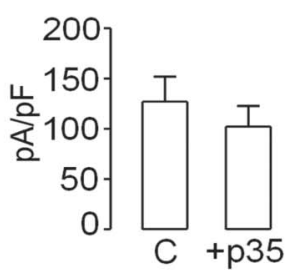

Figure 8. Activation or inhibition of Cdk5 activity regulates P2X2aR-mediated currents in cell lines. (A) Representative recordings evoked by $100 \mu \mathrm{M}$ ATP on PC12 cells in the absence (black tracing), after 6 hours (gray tracing), or after 24 hours (red tracing) of incubation with $30 \mu \mathrm{M}$ roscovitine. Membrane voltage was held at $-60 \mathrm{mV}$. (B) Summary of desensitization constants $\left(\tau_{\text {des }}\right)$ calculated for PC12 cells in control conditions (C) and after 6 and 24 hours of incubation with roscovitine (Rosc). ${ }^{*} P<0.05$, Mann-Whitney test, $n=5$. (C) Summary of current densities obtained in control (C) and roscovitine-treated (Rosc) PC12 cells. $n=5$. (D) Representative recordings of a HEK293 cell expressing the P2X2/3R alone or co-transfected with p35 and the typical slowdesensitizing current (as compared to the fast-desensitizing P2X3R-mediated current) evoked by $10 \mu \mathrm{M} \alpha, \beta$-meATP. (E) Summary of the desensitization constants $\left(\tau_{\text {des }}\right)$ of the currents mediated by the P2X2/3R alone $(C)$ or co-transfected with p35 $\left(+\right.$ p35). $n=4$ to 8 ; ${ }^{*} P<0.05$, Mann-Whitney test. (F) Summary of current densities obtained in the absence $(C)$ and in the presence of p35 (+p35) in P2X2a/3R-expressing HEK293 cells. $n=3$ to 5 . ATP, adenosine triphosphate; Cdk5, cyclin-dependent kinase 5.

roscovitine (20 $\mu \mathrm{M}, 6$ hours), we observed a greater number of fast spike responses (Fig. 9F) that resulted in a decrease of the decay constant (Fig. 9G) without affecting the percentage of responding cells (Fig. 9H) nor the relative amplitude (Fig. 9I) of the $\alpha, \beta$-meATP-evoked responses.

\section{Discussion}

In the present study, we showed a functional interaction between the P2X2aR and the Cdk5/p35 complex. Moreover, the currents mediated by this channel are regulated by the activation of Cdk5, which prevents the development of UDD in whole-cell patchclamp recordings. We suggest that the phosphorylation of the intracellular P2X2aR residue Thr372 by Cdk5/p35 delays the receptor desensitization and such modulation can be physiologically important, especially in pain signaling pathways where both proteins are interacting, as our experiments in PC12 cells and TG neurons strongly suggest.

Phosphorylation and dephosphorylation are common processes that regulate diverse protein functions, including the gating of plasma membrane channels; a number of reviews have summarized the effects of phosphorylation in voltagegated channels and in NMDA, GABA, TRP, and ACh receptors. ${ }^{10,37,45,50}$ The $\mathrm{P} 2 \mathrm{XR}$ gating is also modulated by phosphorylation and dephosphorylation. For example, it has been suggested that PKA regulates P2X2R and P2X3R function, inhibiting the currents mediated by the former and potentiating the latter. ${ }^{16,59}$ In the case of the P2X4R, PKA seems to regulate the receptor trafficking through an accessory protein. ${ }^{6}$ PKC has also been suggested as a regulator of P2XR activity, an effect that for the P2X1R can be mediated by the activation of G-protein-coupled receptors. ${ }^{1}$ Additionally, there is evidence suggesting an effect of PKC on P2X3R. ${ }^{43} \mathrm{~A}$ recent study has shown that calmodulin kinase II can regulate the P2X3R trafficking from and to the plasma membrane. ${ }^{15} \mathrm{Src}$ tyrosine kinase has also been suggested to regulate the
P2X3R, decreasing the receptor-mediated currents through the intracellular Tyr393. ${ }^{17}$

Cyclin-dependent kinase 5 regulates TRPV1 channel function by direct phosphorylation at Thr407, increasing the $\mathrm{Ca}^{2+}$-influx mediated by this channel ${ }^{25,33,40,47}$ and by phosphorylation of kinesin 13B (KIF13B) motor that increases TRPV1 trafficking to the plasma membrane. ${ }^{61}$ In this context, the results showed here are in agreement with previous Cdk5 mechanism of action in the pathways of pain signaling. ${ }^{52}$ Thus, Cdk5-mediated P2X2aR phosphorylation on Thr372 will modify the degree of desensitization of the channels that will finally result in an increased depolarization and increased of $\mathrm{Ca}^{2+}$ influx in the nociceptive neurons. Contrary to TRPV1 and P2X2aR, Cdk5 activation seems to downregulate P2X3R function through the serine phosphorylation of an unidentified accessory protein. ${ }^{38}$ This could reflect a differential regulation of homomeric P2X3Rs and heteromeric $\mathrm{P} 2 \mathrm{X} 2 / 3 \mathrm{Rs}$, that are the subtypes mainly expressed in nociceptive neurons; future functional experiments in nociceptive DRG or TG neurons will help to clarify the role of Cdk5 regulation in these 2 P2XR channel subtypes.

In the present study, our results support a direct interaction of the P2X2aR with Cdk5 and p35. Co-immunolocalization experiments showed a marked overlap between P2X2aR and Cdk5/ p35 signals in both heterologous systems and endogenous protein levels as we found in mouse TG and DRG neurons; this was further supported by co-immunoprecipitation experiments, suggesting that P2X2aR can interact with both Cdk5 and p35. Additionally, we also found that P2X2aR and Cdk5/p35 not only are physically close, but also that their interaction increases threonine phosphorylation. Moreover, the Cdk5 consensus motif ${ }^{372} \mathrm{TPKH}^{375}$ is only present in the rat P2X2aR but not in the splice variant $\mathrm{P} 2 \mathrm{X} 2 \mathrm{bR}$. We identified this sequence as part of the canonical consensus sequence $(\mathrm{S} / \mathrm{T}) \mathrm{PX}(\mathrm{K} / \mathrm{H} / \mathrm{R})$ present in target proteins that are phosphorylated by $\mathrm{Cdk} 5,{ }^{2}$ and we confirmed that this sequence can be a phosphorylation target by the synthesis of peptides containing this sequence and the 

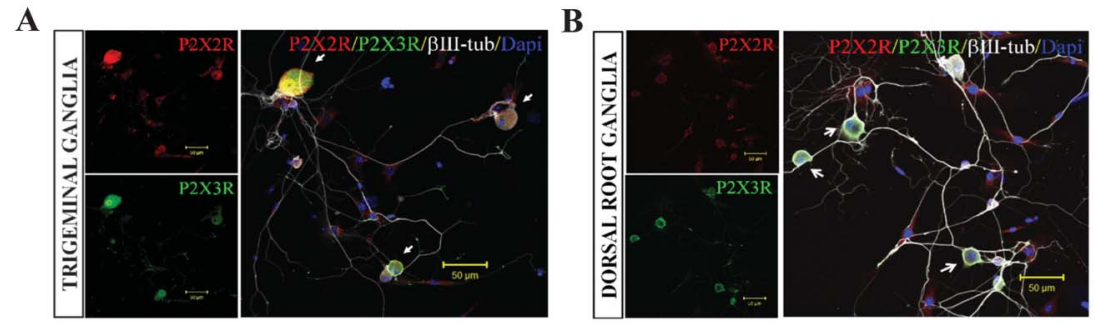

C

TG neurons

D
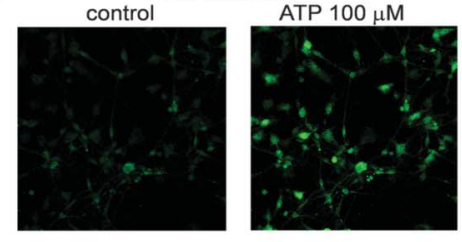

F

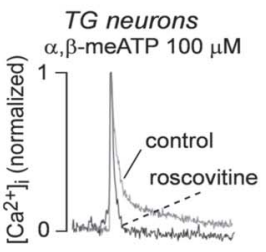

G

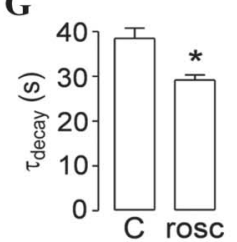

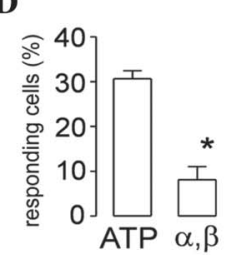

H

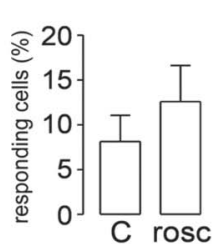

E TG neurons

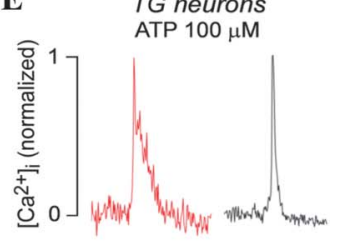

I

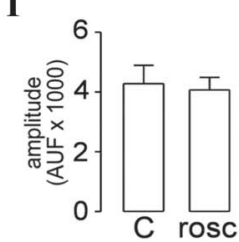

Figure 9. Cyclin-dependent kinase 5 (Cdk5) regulation of the heteromeric P2X2/3Rs. Confocal immunofluorescence of primary cultured neurons of 2 DIV from mouse TG (A) and DRG (B), expressing endogenous levels of P2X2R (red) and P2X3R (green). Neurons were immunostained with $\beta$ III-tubulin (white), and Dapi was used as nuclear staining. Arrows indicate neurons expressing heteromeric P2X2/3R subtype. (C) Representative fields of cultured rat TG neurons loaded with Fluo4 before (left image) and after (right image) the addition of $100 \mu \mathrm{M}$ ATP. (D) Summary of the percentage of responding cells to ATP and $\alpha, \beta-$ meATP $(\alpha, \beta)$. $\mathrm{n}=3$, ${ }^{\star} P$ $<0.05$, Student $t$ test. (E) Typical $\mathrm{Ca}^{2+}$ spikes induced by $100 \mu \mathrm{M}$ ATP. (F) Representative $\mathrm{Ca}^{2+}$ spikes induced by $100 \mu \mathrm{M} \alpha, \beta$-meATP without (control) or with a 6-hour roscovitine (20 $\mathrm{MM}$ ) treatment (rosc). (G-I) Summary of the results obtained in TG neurons without (C) or with the roscovitine treatment (rosc) for decay constant (G), percentage of responding cells $(\mathrm{H})$ and relative amplitude (I). $n=20$ to 71 ; ${ }^{\star} P<0.05$, Mann-Whitney test. ATP, adenosine triphosphate; DRG, dorsal root ganglion; TG, trigeminal ganglion.

homologous Cdk5 consensus motifs present in human, rat, and mouse P2X2aRs; all of these peptides were phosphorylated by the Cdk5/p35 complex, indicating that they can effectively reflect a phosphorylation site in the P2X2aR C-terminal domain.

We tested if the Cdk5-mediated phosphorylation of the P2X2aR could have functional consequences in the activity of this channel, by means of electrophysiological recordings. We found that the activation of endogenous Cdk5, by the expression of its activator p35, significantly prevented P2X2aR desensitization after repetitive ATP applications. We have previously shown that the P2X2aR increases its desensitization rates after successive ATP applications, arising a $\mathrm{Ca}^{2+}$-dependent process termed UDD. ${ }^{14,27}$ The most plausible explanation for this phenomenon is that Cdk5 phosphorylation favors a nondesensitized phenotype and protects the receptor for the $\mathrm{Ca}^{2+}$ -dependent UDD. We have previously showed that UDD requires the presence of $\mathrm{Ca}^{2+}$ in a nanomolar or less concentration to be observed in whole-cell recordings, ${ }^{14}$ and this could reflect the loss of some relevant cytosolic factor to determine P2X2aR desensitization. This can explain the differences observed in the recordings performed in HEK293 cells and Xenopus oocytes, although future experiments will help to completely clarify the mechanism of P2X2aR desensitization. When experiments were performed in Xenopus oocytes, a recording system that preserves the intracellular components, we also observed a prevention of P2X2aR increase in desensitization after Cdk5 activation and this effect was blocked by the Cdk5 inhibitor roscovitine, although desensitization was remarkably smaller than in whole-cell patch-clamp recordings. Moreover, the mutation of T372A prevented the development of UDD in whole-cell recordings in HEK293 cells and inhibited the increase of desensitization in 2-electrode voltage-clamp recordings in oocytes, suggesting the critical role of this residue in this phenomenon. Although we initially expected that this mutant would render more desensitized profiles of currents, we hypothesize that the mutation induced a structural change that probably resulted in this particular phenotype. Although the T372A mutation obliterated the increases in receptor desensitization regardless of the expression system used, it is obvious that the desensitization observed varied depending on the cell host, showing a higher desensitization profile in HEK293 cells as compared to PC12 cells and Xenopus oocytes. We infer that these differences could be the result of particular features of each expression system, but despite that, we confirm that the mutant T372A preserved most of the features characteristic of the $\mathrm{P} 2 \mathrm{X} 2 \mathrm{aR}$, supporting our findings in the present work.

Remarkably, the experiments in PC12 cells and in primary cultures of TG neurons strongly suggest that Cdk5-mediated regulation of the $\mathrm{P} 2 \mathrm{X} 2 \mathrm{R}$ could be physiologically relevant. $\mathrm{PC} 12$ cells endogenously express P2X2R, Cdk5, and p354,23,53; we inferred that if that being the case, the P2X2R-mediated currents are basally phosphorylated by Cdk5, rendering currents with slow desensitization kinetics. As expected, Cdk5 inhibition by roscovitine accelerated the desensitization of P2X2R-mediated currents, in agreement with the results obtained in heterologous systems. Finally, we studied the effects of Cdk5 phosphorylation on the heteromeric P2X2/3R that is the main purinergic receptor involved in pain transmission. ${ }^{31}$ Currently, there is no published 
information if all the splice P2X2R variants can indeed form heteromeric receptors, but we have preliminary data that at least rat $\mathrm{P} 2 \mathrm{X} 2 \mathrm{bR}$ is able to form functional heteromeric channels (Dr. Stanko Stojllkovic, June 2013, oral communication). As the homomeric P2X2aR, the desensitization properties of the P2X2/ 3R were affected by Cdk5 phosphorylation as demonstrated by both electrophysiology of heterologous expressed P2X2/3Rs in HEK293 cells and by $\mathrm{Ca}^{2+}$ imaging of endogenous P2X2/3Rs from primary cultures of TG neurons that also express endogenously p35 and Cdk5. These results strongly suggest a physiological relevance of Cdk5 regulation of purinergic receptors, especially in pain transduction. P2X2/3Rs are commonly formed by 2 P2X3 subunits and 1 P2X2 subunit ${ }^{26}$; however, a recent article has shown that the $\mathrm{P} 2 \mathrm{X}(\mathrm{P} 2 \mathrm{X} 2)_{2}$ stoichiometry also results in functional channels ${ }^{29}$; we infer that these receptors will be even more susceptible to Cdk5 regulation.

In general, the effects of phosphorylation on membrane receptors and channels can be exerted through direct regulation of the channel gating properties, resulting in a greater or smaller conductance or in a change of voltage dependence or in the agonist's $\mathrm{EC}_{50}$, as it has been observed for Kv2.1 or TRPV1 channels. ${ }^{36,42}$ Other described effects of channel phosphorylation o dephosphorylation are channel internalization and/or changes in trafficking and degradation, as for example, AMPA and NMDA glutamate receptors. ${ }^{24,32}$ Interestingly, it has also been reported that a phosphorylation and dephosphorylation can regulate the desensitization of NMDA receptors, by regulating the number of functional channels in the membrane. ${ }^{58}$ In our study, it seems that Cdk5 phosphorylation does not affect the number of functional channels (it has no effect on current amplitudes), but rather it affects the desensitization properties by changing its gating properties because we did not observe changes in the maximal currents nor in ATP $\mathrm{EC}_{50}$ after Cdk5 activation. Accordingly, we did not observe a significant increase in the protein levels of the P2X2aR in immunoblots when the receptor was co-expressed with p35 or Cdk5; however, future studies will be conducted to explore P2X2aR trafficking to the plasma membrane when Cdk5 is highly activated. There is evidence showing that Cdk5 regulate the activation of several ion channels and membrane receptors, suggesting a role during peripheral and central sensitization in pain pathways. ${ }^{21,47,52,60}$ For example, Cdk5 can phosphorylate NMDA receptors at Ser1232 of the NR2A subunit ${ }^{32}$ and regulate its surface expression through NR2B subunit phosphorylation. ${ }^{44} \mathrm{P} / \mathrm{Q}$-type voltage-dependent $\mathrm{Ca}^{2+}$ channels are also targets for Cdk5 phosphorylation, a process that regulates neurotransmitter release. ${ }^{51}$

In summary, we have identified a novel mechanism of regulation of $\mathrm{P} 2 \mathrm{X} 2 \mathrm{R}$ desensitization by Cdk5-mediated phosphorylation, and we have presented evidence that this regulation could be relevant in pain signaling, such as P2X2/3R-mediated responses in TG neurons. Future efforts should be focused on characterizing the mechanism of $\mathrm{P} 2 \mathrm{X} 2 \mathrm{aR}$ desensitization and whether phosphorylation and $\mathrm{Ca}^{2+}$-mediated effects are related. Our findings suggest that the P2X2aR is a novel substrate for Cdk5-mediated phosphorylation, which might play an important role in the physiological processes such as pain signaling and therefore could be a potential novel target for developing pain therapies.

\section{Conflict of interest statement}

The authors have no conflict of interest to declare.

Supported by FONDECYT 11121302, 1161490, and FONDEQUIP EQM140100 (to C.C.); FONDECYT 1151043 (to E.U.); FONDECYT 1140325 and FONDAP 15150012 (to C.G.-B.), the Intramural Research Programs of Eunice Kennedy Shiver National Institute of Child Health and Human Development (S.S.S., C.C.), and the National Institute of Dental and Craniofacial Research (B.H., A.T. and A.B.K.), the National Institutes of Health.

\section{Acknowledgements}

The authors would like to thank Drs Harish Pant and Niranjana Amin for their guidance in measuring Cdk5 activity and Dr Michaela Prochazkova for helpful discussion. C. Coddou and E. Utreras contributed equally to this work.

\section{Article history:}

Received 2 May 2017

Received in revised form 26 June 2017

Accepted 6 July 2017

Available online 17 July 2017

\section{References}

[1] Ase AR, Raouf R, Belanger D, Hamel E, Seguela P. Potentiation of P2X1 ATP-gated currents by 5 -hydroxytryptamine $2 \mathrm{~A}$ receptors involves diacylglycerol-dependent kinases and intracellular calcium. J Pharmacol Exp Ther 2005;315:144-54.

[2] Borquez DA, Olmos C, Alvarez S, Di Genova A, Maass A, GonzalezBillault C. Bioinformatic survey for new physiological substrates of Cyclindependent kinase 5. Genomics 2013;101:221-8.

[3] Boue-Grabot E, Archambault V, Seguela P. A protein kinase C site highly conserved in P2X subunits controls the desensitization kinetics of P2X(2) ATP-gated channels. J Biol Chem 2000;275:10190-5.

[4] Brake AJ, Wagenbach MJ, Julius D. New structural motif for ligand-gated ion channels defined by an ionotropic ATP receptor. Nature 1994;371:519-23.

[5] Brandle U, Spielmanns P, Osteroth R, Sim J, Surprenant A, Buell G, Ruppersberg JP, Plinkert PK, Zenner HP, Glowatzki E. Desensitization of the P2X(2) receptor controlled by alternative splicing. FEBS Lett 1997; 404:294-8.

[6] Brown DA, Yule DI. Protein kinase A regulation of P2X(4) receptors: requirement for a specific motif in the $\mathrm{C}$-terminus. Biochim Biophys Acta 2010;1803:275-87.

[7] Burnstock G. Physiology and pathophysiology of purinergic neurotransmission. Physiol Rev 2007;87:659-797.

[8] Burnstock G. Purinergic mechanosensory transduction and visceral pain. Mol Pain 2009;5:69.

[9] Burnstock G. Purinergic mechanisms and pain. Adv Pharmacol 2016;75: 91-137.

[10] Cerda O, Baek JH, Trimmer JS. Mining recent brain proteomic databases for ion channel phosphosite nuggets. J Gen Physiol 2011;137:3-16.

[11] Clyne JD, LaPointe LD, Hume RI. The role of histidine residues in modulation of the rat P2X(2) purinoceptor by zinc and pH. J Physiol 2002; 539:347-59.

[12] Coddou C, Codocedo JF, Li S, Lillo JG, Acuna-Castillo C, Bull P, Stojilkovic SS, Huidobro-Toro JP. Reactive oxygen species potentiate the P2X2 receptor activity through intracellular Cys430. J Neurosci 2009;29: 12284-91.

[13] Coddou C, Yan Z, Obsil T, Huidobro-Toro JP, Stojilkovic SS. Activation and regulation of purinergic P2X receptor channels. Pharmacol Rev 2011; 63:641-83.

[14] Coddou C, Yan Z, Stojilkovic SS. Role of domain calcium in purinergic P2X2 receptor channel desensitization. Am J Physiol Cell Physiol 2015; 308:C729-736.

[15] Chen XQ, Zhu JX, Wang Y, Zhang X, Bao L. CaMKIlalpha and caveolin-1 cooperate to drive ATP-induced membrane delivery of the P2X3 receptor. J Mol Cell Biol 2014;6:140-53.

[16] Chow YW, Wang HL. Functional modulation of P2X2 receptors by cyclic AMP-dependent protein kinase. J Neurochem 1998;70:2606-12.

[17] D'Arco M, Giniatullin R, Leone V, Carloni P, Birsa N, Nair A, Nistri A, Fabbretti E. The C-terminal Src inhibitory kinase (Csk)-mediated tyrosine phosphorylation is a novel molecular mechanism to limit P2X3 receptor function in mouse sensory neurons. J Biol Chem 2009; 284:21393-401.

[18] De Roo M, Boue-Grabot E, Schlichter R. Selective potentiation of homomeric P2X2 ionotropic ATP receptors by a fast non-genomic action of progesterone. Neuropharmacology 2010;58:569-77. 
[19] Dhariwala FA, Rajadhyaksha MS. An unusual member of the Cdk family: Cdk5. Cell Mol Neurobiol 2008;28:351-69.

[20] Dhavan R, Tsai LH. A decade of CDK5. Nat Rev Mol Cell Biol 2001;2: 749-59.

[21] Fang H, Zhang HH, Yang BX, Huang JL, Shun JL, Kong FJ, Peng X, Chen ZG, Lu JM. Cdk5 contributes to inflammation-induced thermal hyperalgesia mediated by the p38 MAPK pathway in microglia. Brain Res 2015;1619:166-75.

[22] Fujiwara Y, Kubo Y. Regulation of the desensitization and ion selectivity of ATP-gated P2X2 channels by phosphoinositides. J Physiol 2006;576: 135-49.

[23] Harada T, Morooka T, Ogawa S, Nishida E. ERK induces p35, a neuronspecific activator of Cdk5, through induction of Egr1. Nat Cel Biol 2001;3: 453-9.

[24] Hawasli AH, Benavides DR, Nguyen C, Kansy JW, Hayashi K, Chambon P, Greengard P, Powell CM, Cooper DC, Bibb JA. Cyclin-dependent kinase 5 governs learning and synaptic plasticity via control of NMDAR degradation. Nat Neurosci 2007;10:880-6.

[25] Jendryke T, Prochazkova M, Hall BE, Nordmann GC, Schladt M, Milenkovic VM, Kulkarni AB, Wetzel CH. TRPV1 function is modulated by Cdk5-mediated phosphorylation: insights into the molecular mechanism of nociception. Scientific Rep 2016;6:22007.

[26] Jiang LH, Kim M, Spelta V, Bo X, Surprenant A, North RA. Subunit arrangement in P2X receptors. J Neurosci 2003;23:8903-10.

[27] Khadra A, Yan Z, Coddou C, Tomic M, Sherman A, Stojilkovic SS. Gating properties of the P2X2a and P2X2b receptor channels: experiments and mathematical modeling. J Gen Physiol 2012;139:333-48.

[28] Koshimizu T, Tomic M, Koshimizu M, Stojilkovic SS. Identification of amino acid residues contributing to desensitization of the P2X2 receptor channel. J Biol Chem 1998;273:12853-7.

[29] Kowalski M, Hausmann R, Schmid J, Dopychai A, Stephan G, Tang Y, Schmalzing G, Illes P, Rubini P. Flexible subunit stoichiometry of functional human P2X2/3 heteromeric receptors. Neuropharmacology 2015;99:115-30.

[30] Lew J, Huang QQ, Qi Z, Winkfein RJ, Aebersold R, Hunt T, Wang JH. A brain-specific activator of cyclin-dependent kinase 5. Nature 1994;371: 423-6.

[31] Lewis C, Neidhart S, Holy C, North RA, Buell G, Surprenant A. Coexpression of $\mathrm{P} 2 \mathrm{X} 2$ and $\mathrm{P} 2 \mathrm{X} 3$ receptor subunits can account for ATP-gated currents in sensory neurons. Nature 1995;377:432-5.

[32] Li BS, Sun MK, Zhang L, Takahashi S, Ma W, Vinade L, Kulkarni AB, Brady RO, Pant HC. Regulation of NMDA receptors by cyclin-dependent kinase-5. Proc Natl Acad Sci USA 2001;98:12742-7.

[33] Liu J, Du J, Yang Y, Wang Y. Phosphorylation of TRPV1 by cyclindependent kinase 5 promotes TRPV1 surface localization, leading to inflammatory thermal hyperalgesia. Exp Neurol 2015;273:253-62.

[34] Lorca RA, Coddou C, Gazitua MC, Bull P, Arredondo C, Huidobro-Toro JP. Extracellular histidine residues identify common structural determinants in the copper/zinc P2X2 receptor modulation. J Neurochem 2005;95: 499-512.

[35] Mohapatra DP, Nau C. Desensitization of capsaicin-activated currents in the vanilloid receptor TRPV1 is decreased by the cyclic AMP-dependent protein kinase pathway. J Biol Chem 2003;278:50080-90.

[36] Mohapatra DP, Nau C. Regulation of Ca2 +-dependent desensitization in the vanilloid receptor TRPV1 by calcineurin and CAMP-dependent protein kinase. J Biol Chem 2005;280:13424-32.

[37] Moss SJ, Smart TG. Modulation of amino acid-gated ion channels by protein phosphorylation. Int Rev Neurobiol 1996;39:1-52.

[38] Nair A, Simonetti M, Fabbretti E, Nistri A. The Cdk5 kinase downregulates ATP-gated ionotropic P2X3 receptor function via serine phosphorylation. Cell Mol Neurobiol 2010;30:505-9.

[39] Newbolt A, Stoop R, Virginio C, Surprenant A, North RA, Buell G, Rassendren F. Membrane topology of an ATP-gated ion channel (P2X receptor). J Biol Chem 1998;273:15177-82.

[40] Pareek TK, Keller J, Kesavapany S, Agarwal N, Kuner R, Pant HC, ladarola MJ, Brady RO, Kulkarni AB. Cyclin-dependent kinase 5 modulates nociceptive signaling through direct phosphorylation of transient receptor potential vanilloid 1. Proc Natl Acad Sci USA 2007; 104:660-5.

[41] Pareek TK, Kulkarni AB. Cdk5: a new player in pain signaling. Cell Cycle 2006:5:585-8.

[42] Park KS, Mohapatra DP, Misonou H, Trimmer JS. Graded regulation of the Kv2.1 potassium channel by variable phosphorylation. Science 2006; 313:976-9.

[43] Paukert M, Osteroth R, Geisler HS, Brandle U, Glowatzki E, Ruppersberg JP, Grunder S. Inflammatory mediators potentiate ATP-gated channels through the P2X(3) subunit. J Biol Chem 2001;276:21077-82.

[44] Peng HY, Chen GD, Tung KC, Lai CY, Hsien MC, Chiu CH, Lu HT, Liao JM, Lee SD, Lin TB. Colon mustard oil instillation induced cross-organ reflex sensitization on the pelvic-urethra reflex activity in rats. PAIN 2009; 142:75-88.

[45] Pingle SC, Matta JA, Ahern GP. Capsaicin receptor: TRPV1 a promiscuous TRP channel. Handb Exp Pharmacol 2007;79:155-71.

[46] Prochazkova M, Terse A, Amin ND, Hall B, Utreras E, Pant HC, Kulkarni AB. Activation of cyclin-dependent kinase 5 mediates orofacial mechanical hyperalgesia. Mol Pain 2013;9:66.

[47] Rozas P, Lazcano P, Pina R, Cho A, Terse A, Pertusa M, Madrid R, Gonzalez-Billault C, Kulkarni AB, Utreras E. Targeted overexpression of tumor necrosis factor-alpha increases cyclin-dependent kinase 5 activity and TRPV1-dependent Ca2 + influx in trigeminal neurons. PAIN 2016; 157:1346-62.

[48] Simon J, Kidd EJ, Smith FM, Chessell IP, Murrell-Lagnado R, Humphrey PP, Barnard EA. Localization and functional expression of splice variants of the P2X2 receptor. Mol Pharmacol 1997;52:237-48.

[49] Surprenant A, North RA. Signaling at purinergic P2X receptors. Annu Rev Physiol 2009;71:333-59.

[50] Swope SL, Moss SJ, Raymond LA, Huganir RL. Regulation of ligandgated ion channels by protein phosphorylation. Adv Second Messenger Phosphoprotein Res 1999;33:49-78.

[51] Tomizawa K, Ohta J, Matsushita M, Moriwaki A, Li ST, Takei K, Matsui H. Cdk5/p35 regulates neurotransmitter release through phosphorylation and downregulation of $\mathrm{P} / \mathrm{Q}$-type voltage-dependent calcium channel activity. J Neurosci 2002;22:2590-7.

[52] Utreras E, Futatsugi A, Pareek TK, Kulkarni AB. Molecular roles of Cdk5 in pain signaling. Drug Discov Today Ther Strateg 2009;6:105-11.

[53] Utreras E, Futatsugi A, Rudrabhatla P, Keller J, ladarola MJ, Pant HC, Kulkarni AB. Tumor necrosis factor-alpha regulates cyclin-dependent kinase 5 activity during pain signaling through transcriptional activation of p35. J Biol Chem 2009;284:2275-84.

[54] Utreras E, Keller J, Terse A, Prochazkova M, ladarola MJ, Kulkarni AB. Transforming growth factor-beta1 regulates Cdk5 activity in primary sensory neurons. J Biol Chem 2012;287:16917-29.

[55] Utreras E, Maccioni R, Gonzalez-Billault C. Cyclin-dependent kinase 5 activator p35 over-expression and amyloid beta synergism increase apoptosis in cultured neuronal cells. Neuroscience 2009;161:978-87.

[56] Utreras E, Prochazkova M, Terse A, Gross J, Keller J, ladarola MJ, Kulkarni AB. TGF-beta1 sensitizes TRPV1 through Cdk5 signaling in odontoblast-like cells. Mol Pain 2013;9:24.

[57] Utreras E, Terse A, Keller J, ladarola MJ, Kulkarni AB. Resveratrol inhibits Cdk5 activity through regulation of p35 expression. Mol Pain 2011;7:49.

[58] Vissel B, Krupp JJ, Heinemann SF, Westbrook GL. A use-dependent tyrosine dephosphorylation of NMDA receptors is independent of ion flux. Nat Neurosci 2001;4:587-96.

[59] Wang C, Gu Y, Li GW, Huang LY. A critical role of the cAMP sensor Epac in switching protein kinase signalling in prostaglandin E2-induced potentiation of P2X3 receptor currents in inflamed rats. J Physiol 2007; 584:191-203.

[60] Wang GQ, Cen C, Li C, Cao S, Wang N, Zhou Z, Liu XM, Xu Y, Tian NX, Zhang Y, Wang J, Wang LP, Wang Y. Deactivation of excitatory neurons in the prelimbic cortex via Cdk5 promotes pain sensation and anxiety. Nat Commun 2015;6:7660.

[61] Xing BM, Yang YR, Du JX, Chen HJ, Qi C, Huang ZH, Zhang Y, Wang Y. Cyclin-dependent kinase 5 controls TRPV1 membrane trafficking and the heat sensitivity of nociceptors through KIF13B. J Neurosci 2012;32:14709-21. 